
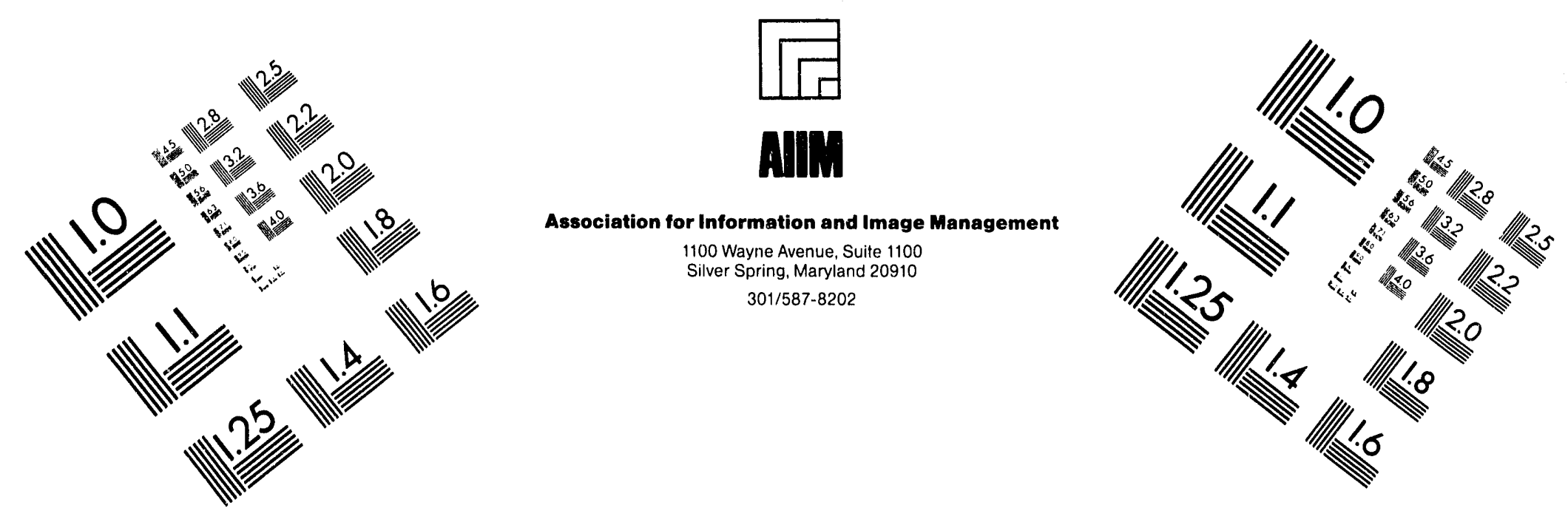

\title{
Centimeter
}

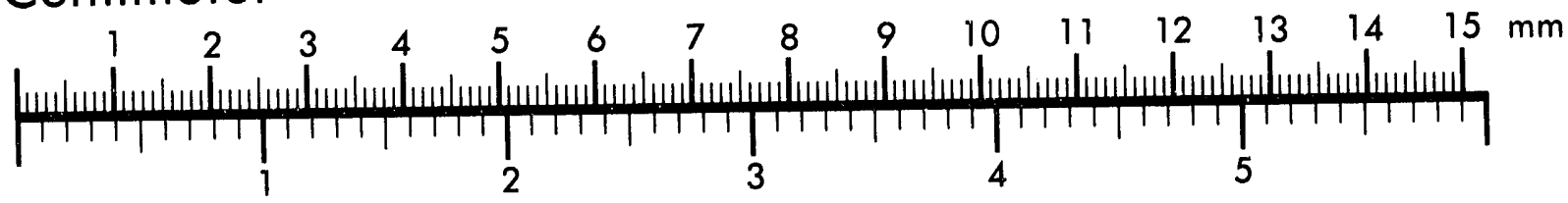

Inches
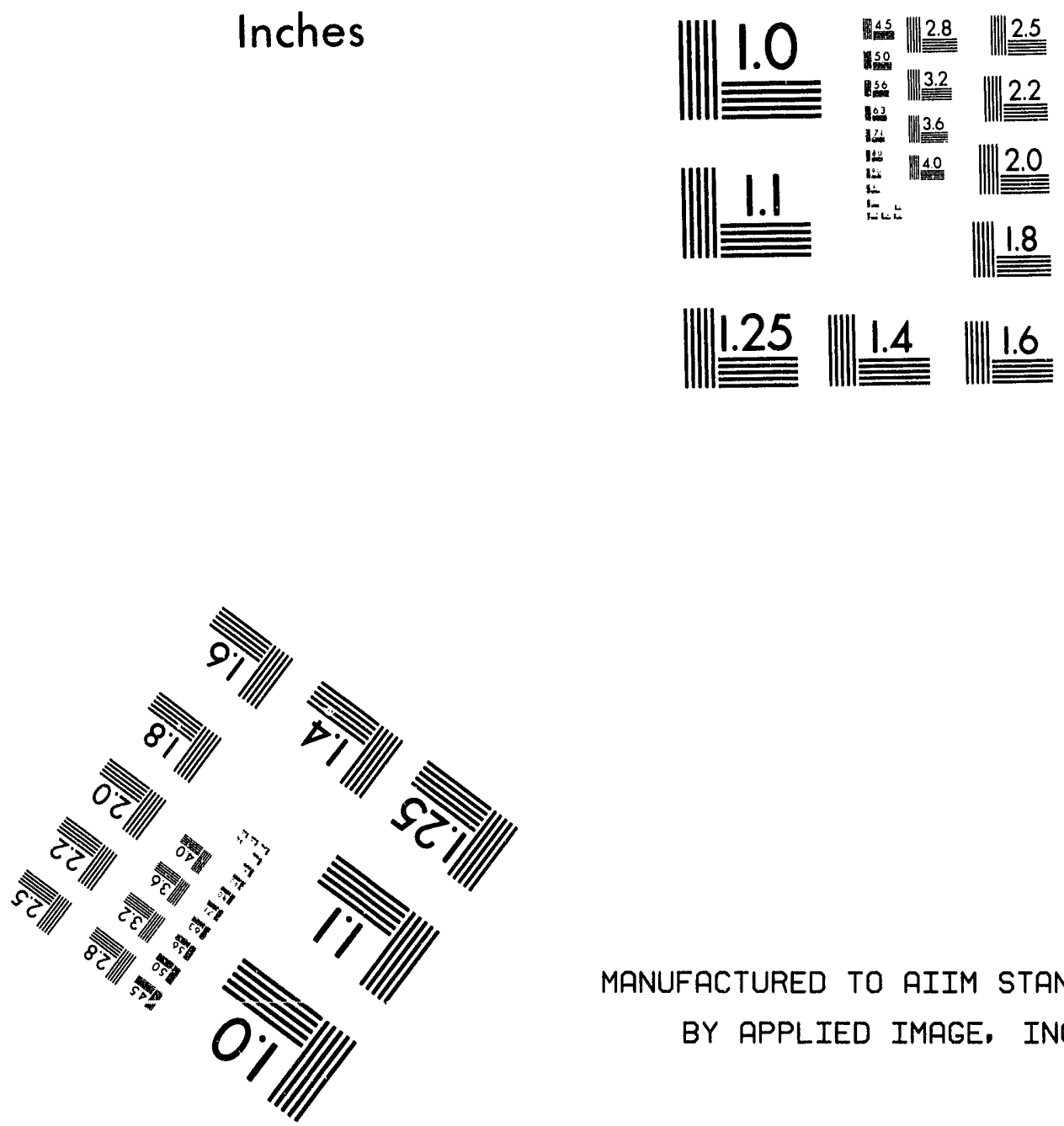

MANUFACTURED TO AIIM STANDARDS

BY APPLIED IMAGE, INC.

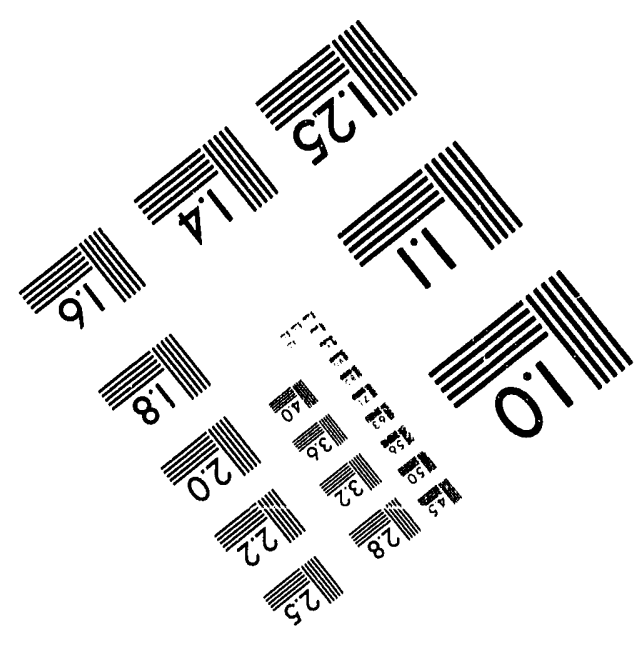



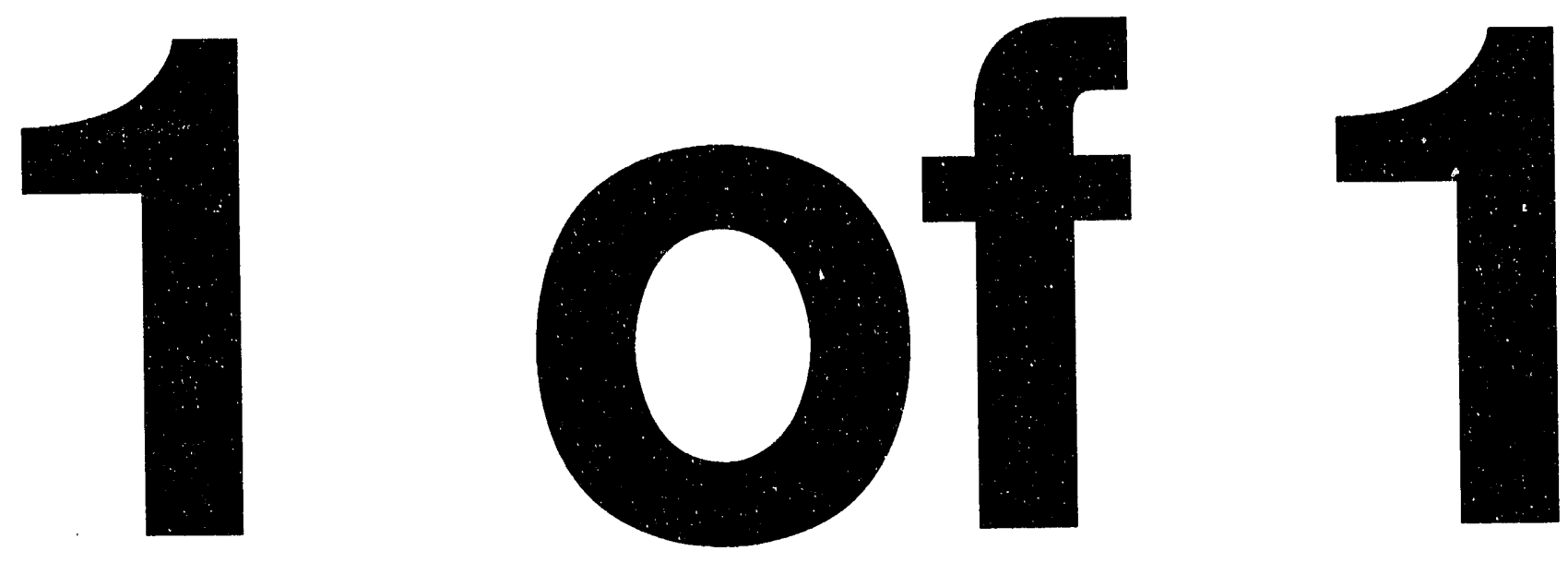
LBL-32165 (Rev. 3)

\title{
Public Census Data on CD-ROM at Lawrence Berkeley Laboratory
}

\author{
by
}

Deane W. Merrill

Information and Computing Sciences Division

Lawrence Berkeley Laboratory

University of California

Berkeley, California 94720

\section{January 1993}

\section{MASTER}

This work was supported by the Director, Office of Epidemiology and Health Surveillance, Office of Health, Office of Environment, Safety and Health, U.S. Department of Energy under Contract No. DE-AC0376 SF00098. 


\section{INTRODUCTION}

The Comprehensive Epidemiologic Data Resource (CEDR) and Populations at Risk to Environmental Pollution (PAREP) projects, of the Information and Computing Sciences Division (ICSD) at Lawrence Berkeley Laboratory (LBL), are using public socio-economic and geographic data files which are available to CEDR and PAREP collaborators via LBL's computing network. At this time $72 \mathrm{CD}$-ROM diskettes (approximately 37 gigabytes) are on line via the Unix file server cedrcd.1bl.gov. Most of the files are from the U.S. Bureau of the Census, and many of these pertain to the 1990 Census of Population and Housing.

All the CD-ROM diskettes contain documentation in the form of ASCII text files. In addition, printed documentation for most files is available for inspection at University of California Data and Technical Assistance (UC DATA), tel. (510) 642-6571, or the UC Documents Library, tel. (510) 642-2569, both located on the UC Berkeley Campus. Many of the CD-ROM diskettes distributed by the Census Bureau contain software for PC compatible computers, for easily accessing the data.

Shared access to the data is maintained through a collaboration among the CEDR and PAREP projects at LBL, and UC DATA, and the UC Documents Library. LBL is grateful to UC DATA and the UC Documents Library for the use of their CD-ROM diskettes. Shared access to LBL facilities may be restricted in the future if costs become prohibitive.

Via the Sun Network File System (NFS), these data can be exported to Internet computers for direct access by the user's application program(s). Due to the size of the files, this access method is preferred over File Transfer Protocol (FTP) access. Please contact Deane Merrill (dwmerrill@lbl.gov) if you wish to make use of the data. 


\section{SUMMARY OF DATA ON CD-ROM}

The table below summarizes data on CD-ROM currently available on line (date = release date of CD-ROM diskette(s); location = path name in cedrcd.lbl.gov; no = number of CD-ROM diskettes; $\mathrm{MB}=$ size in megabytes; $\mathrm{pg}=$ page number in this document.)

Most of the CD-ROM diskettes have their own directory structure, including separate files for documentation and different types of data. For specific information regarding each CD-ROM diskette, consult the page numbers indicated in this table.

Additional technical information is provided at the end of this document.

\begin{tabular}{|c|c|c|c|c|c|c|}
\hline description & date & format & location & no & MB & $\mathrm{pg}$ \\
\hline total & & & & 72 & 37,000 & \\
\hline $\begin{array}{l}1988 \text { County and City } \\
\text { Data Book }\end{array}$ & $6 / 89$ & dBase & /cdroms/disk1 & 1 & 11 & 4 \\
\hline $\begin{array}{l}1986 \text { and } 1987 \text { County } \\
\text { Business Patterns }\end{array}$ & $8 / 90$ & dBase & /cdroms/disk4 & 1 & 337 & 6 \\
\hline $\begin{array}{l}1987 \text { Census of } \\
\text { Agriculture }\end{array}$ & $8 / 90$ & dBase & /cdroms/disk 9 & 1 & 605 & 8 \\
\hline $\begin{array}{l}1987 \text { Economic Censuses: } \\
\text { release } 1 D\end{array}$ & $11 / 91$ & dBase & /cdroms/disk5 & 1 & 431 & 10 \\
\hline $\begin{array}{l}1987 \text { Economic Censuses: } \\
\text { release } 2 \mathrm{~B}\end{array}$ & $3 / 92$ & dBase & /cdroms/disk23 & 1 & 347 & 11 \\
\hline $\begin{array}{l}1969-90 \text { Regional } \\
\text { Economic Information } \\
\text { System (REIS) }\end{array}$ & $5 / 92$ & ASCII & /cdroms/disk92 & 1 & 492 & 12 \\
\hline $\begin{array}{l}\text { 1988-91 Current } \\
\text { Population Survey }\end{array}$ & $\begin{array}{l}8 / 91- \\
10 / 91 \\
\end{array}$ & ASCII & $\begin{array}{l}\text { /cdroms/disk8, } \\
\text { /cdroms/disk24 }\end{array}$ & 2 & 1,123 & 13 \\
\hline $\begin{array}{l}1990 \text { Census: PL94-171 } \\
\text { redistricting data }\end{array}$ & $\begin{array}{l}2 / 91- \\
3 / 91\end{array}$ & dBase & $\begin{array}{l}\text { /cdroms/disk10- } \\
\text { /cdroms/disk19 }\end{array}$ & 10 & $5 \cdot 300$ & 14 \\
\hline $\begin{array}{l}1990 \text { TIGER files: } \\
\text { postcensus release }\end{array}$ & $\begin{array}{l}6 / 91- \\
9 / 91 \\
\end{array}$ & ASCII & $\begin{array}{l}\text { /cdroms/disk25- } \\
\text { /cdroms/disk68 }\end{array}$ & 44 & 24,000 & 18 \\
\hline $\begin{array}{l}1990 \text { Census: Summary } \\
\text { Tape File } 1 \mathrm{~A} \\
\text { (California) }\end{array}$ & $9 / 91$ & dBase & /caroms/disk22 & 1 & 656 & 23 \\
\hline $\begin{array}{l}1990 \text { Census: Summary } \\
\text { Tape File 1C (US } \\
\text { Summary) }\end{array}$ & $11 / 92$ & dBase & /cdroms/disk 89 & 1 & 301 & 25 \\
\hline $\begin{array}{l}1990 \text { Census: Summary } \\
\text { Tape Eile } 3 \mathrm{~A} \text { (Los } \\
\text { Angeles area) }\end{array}$ & $\begin{array}{l}5 / 92- \\
10 / 92\end{array}$ & dBase & $\begin{array}{l}\text { /cdroms/disk69- } \\
\text { /cdroms/disk70 }\end{array}$ & 2 & 1,258 & 27 \\
\hline
\end{tabular}




\begin{tabular}{|c|c|c|c|c|c|c|}
\hline description & date & format & location & no & MB & $\mathrm{pg}$ \\
\hline USA county statistics & $7 / 92$ & dBase & /cdroms/disk90 & 1 & 88 & 29 \\
\hline $\begin{array}{l}\text { Digital Chart of the } \\
\text { World }\end{array}$ & $\begin{array}{l}1 / 92- \\
6 / 92 \\
\end{array}$ & binary & $\begin{array}{l}\text { /cdroms/disk85- } \\
\text { /cdroms/disk88 }\end{array}$ & 4 & 1,587 & 30 \\
\hline $\begin{array}{l}1992 \text { National Health } \\
\text { Interview Survey }\end{array}$ & $6 / 92$ & binary & /cdroms/disk71 & 1 & 171 & 32 \\
\hline
\end{tabular}




\section{COUNTY AND CITY DATA BOOK}

As indicated in Table I, the CD-ROM diskette containing the 1988 County and City Data Book (in dBase format) is:

$$
\text { cedrcd.lbl.gov: / cdroms/diskl }
$$

which is currently exported from:

$$
\text { parep2.1bl.gov: /disk1 }
$$

\section{EXTRACT}

To view the 1988 County and City Data Book data under PC-NFS with the Census Bureau's program EXTRACT, first put the necessary auxiliary files into a directory $c: \backslash c c d b a u x$ as follows. (This is necessary for the 1988 County and City Data Book. Auxiliary files for some other Census data bases are on the CD-ROM diskettes.)

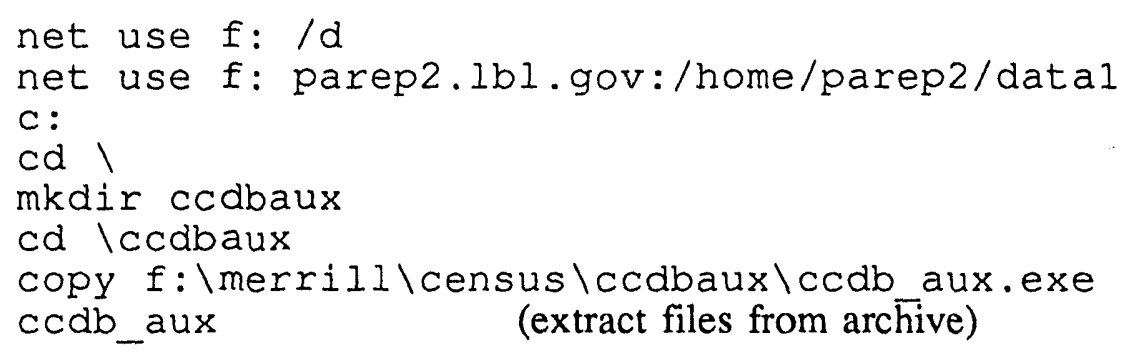

To view the 1988 County and City Data Book data under PC-NFS with EXTRACT, type:

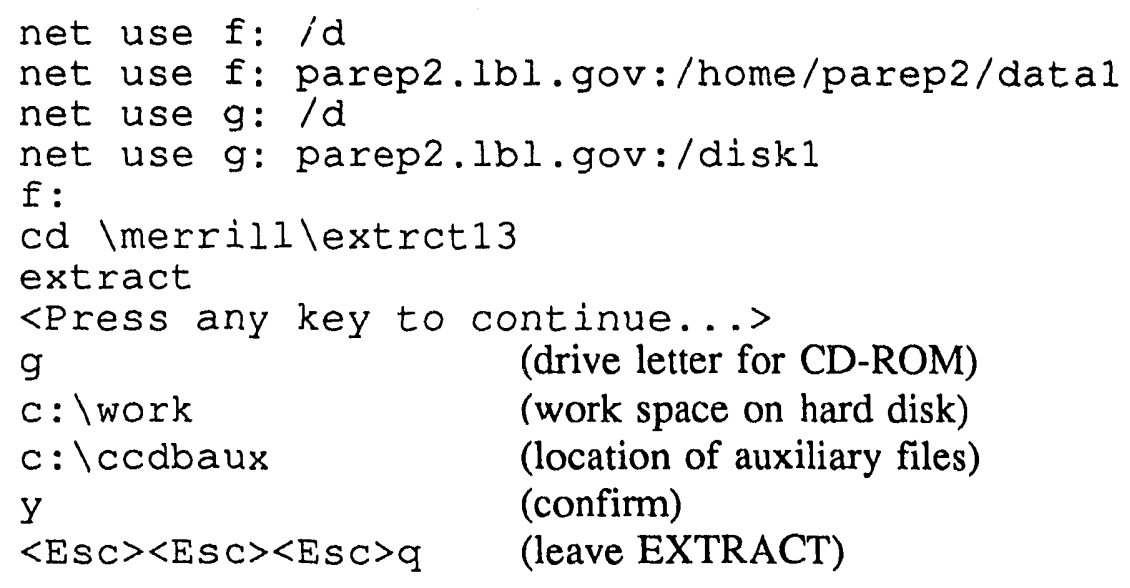


dBASE

To view the 1988 County and City Data Book data under PC-NFS with dBase III+ or dBase IV, type the following:

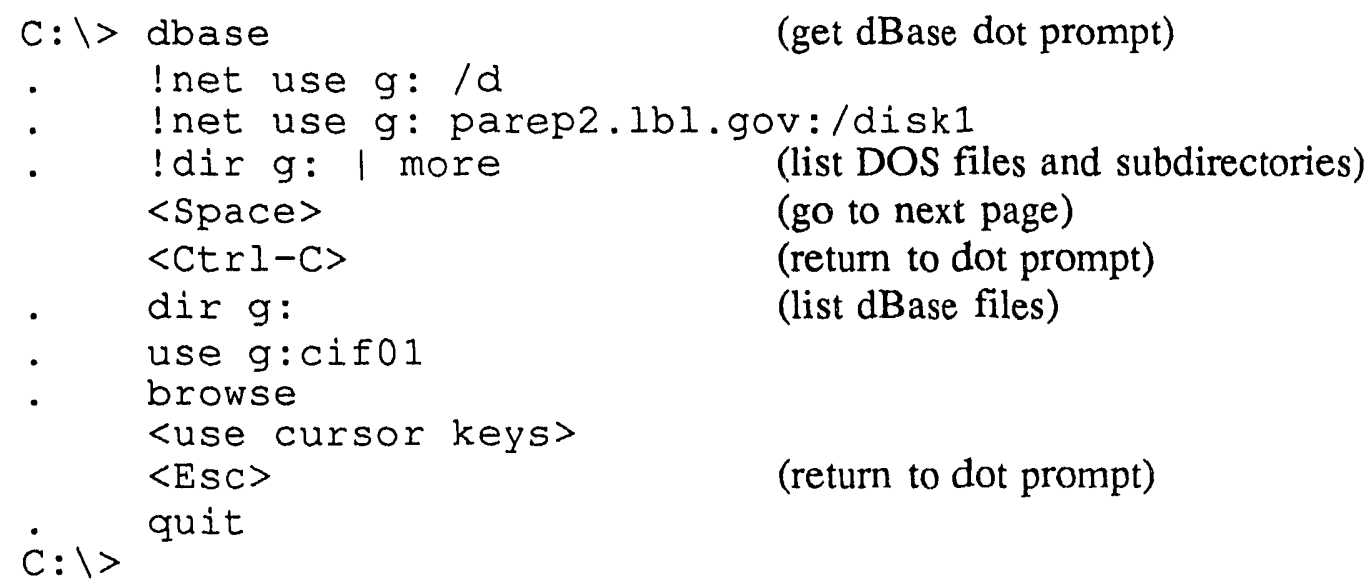




\section{AND 1987 COUNTY BUSINESS PATTERNS}

As indicated in Table I, the CD-ROM diskette containing the 1986 and 1987 County Business Patterns (in dBase format) is:

$$
\text { cedrcd.lbl.gov: / cdroms/disk4 }
$$

which is currently exported from:

$$
\text { parep2.Ibl.gov: /disk4 }
$$

\section{EXTRACT}

To view the 1986 and 1987 County Business Patterns data under PC-NFS with the Census Bureau's program EXTRACT, first put the necessary auxiliary files into a directory c: \cpbauxil as follows. (This is necessary for the 1986 and 1987 County Business Patterns. Auxiliary files for some other Census Bureau data bases are on the CD-ROM diskettes.)

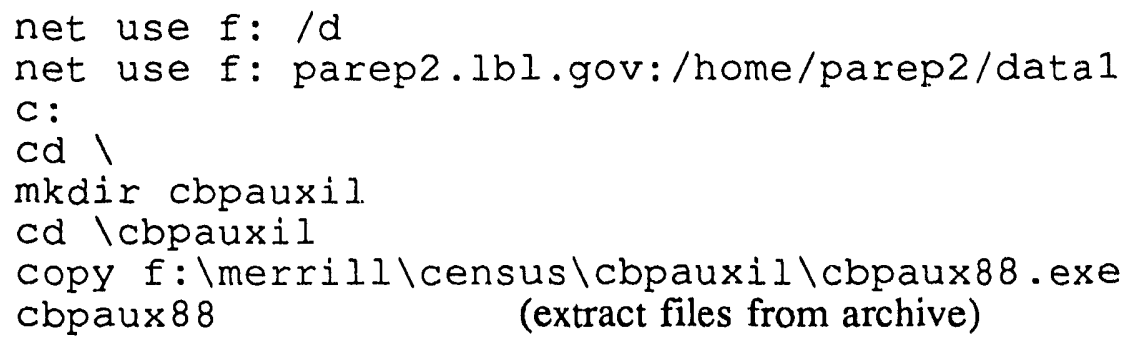

To view the 1986 and 1987 County Business Patterns data under PC-NFS with EXTRACT, type:

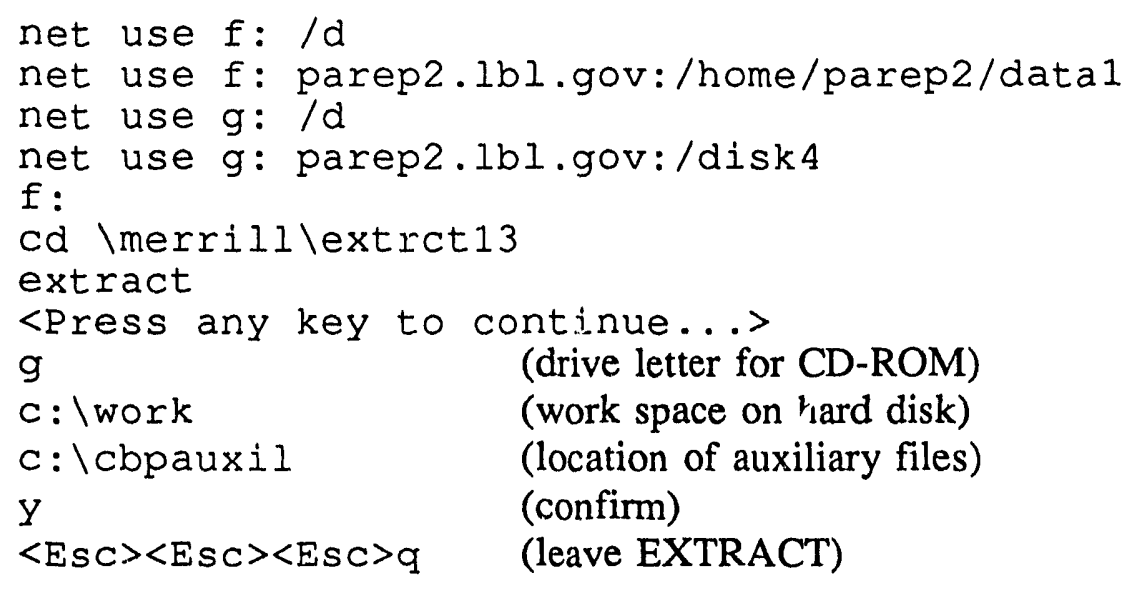




\section{dBASE}

To view (for example) the 1987 California data under PC-NFS with dBase IIIt or dBase IV, type the following:

\begin{tabular}{|c|c|c|}
\hline$C: \backslash>$ & dbase & (get dBase dot prompt) \\
\hline . & !net use $g: / d$ & \\
\hline . & !net use g: parep2.1bl.g & ov: /disk 4 \\
\hline • & !dir g: I more & (list DOS files and subdirectories) \\
\hline & $<$ Space $>$ & (go to next page) \\
\hline & $<\operatorname{Ctr} 1-C>$ & (return to dot prompt) \\
\hline - & !net use $h: / d$ & \\
\hline & !net use $h$ : parep2.1bl.g & ov: /disk4/cbp87 \\
\hline & !dir h: I more & (list DOS files and subdirectories) \\
\hline & $<$ Space $>$ & (go to next page) \\
\hline & $<\operatorname{Ctrl}-\mathrm{C}\rangle$ & (return to dot prompt) \\
\hline • & $\operatorname{dir} h:$ & (list dBase files) \\
\hline & use $h: c b p 87 \mathrm{ca}$ & \\
\hline & browse & \\
\hline & $\begin{array}{l}<\text { use cursor keys } \\
<\text { Esc }>\end{array}$ & (return to dot prompt) \\
\hline 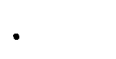 & !more <g: cbpcdrom.doc & (view documentation) \\
\hline & $<$ Space $>$ & (go to next page) \\
\hline & $<\operatorname{Ctrl}-\mathrm{C}\rangle$ & (return to dot prompt) \\
\hline & quit & \\
\hline
\end{tabular}




\section{CENSUS OF AGRICULTURE}

As indicated in Table I, the CD-ROM diskette containing the 1987 Census of Agriculture (in dBase format) is:

cedrcd.Ibl.gov: /cdroms/disk9

which is currently exported from:

parep2.Ibl.gov: /disk9

\section{EXTRACT}

To view the data under PC-NFS with the Census Bureau's program EXTRACT, type the following:

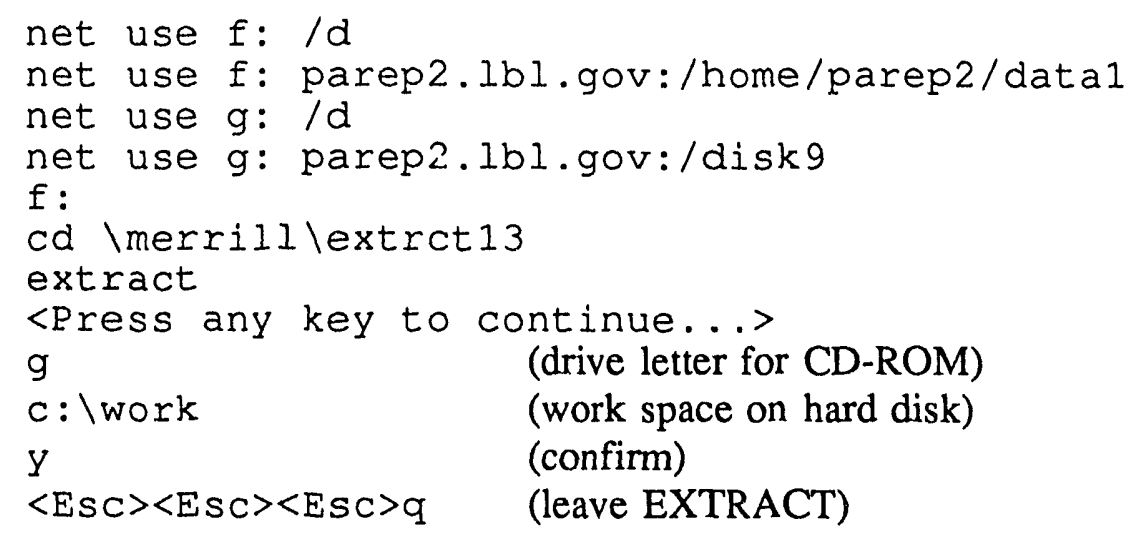


dBASE

To view (for example) the county level California data under PC-NFS with dBase III+ or dBase IV, type the following:

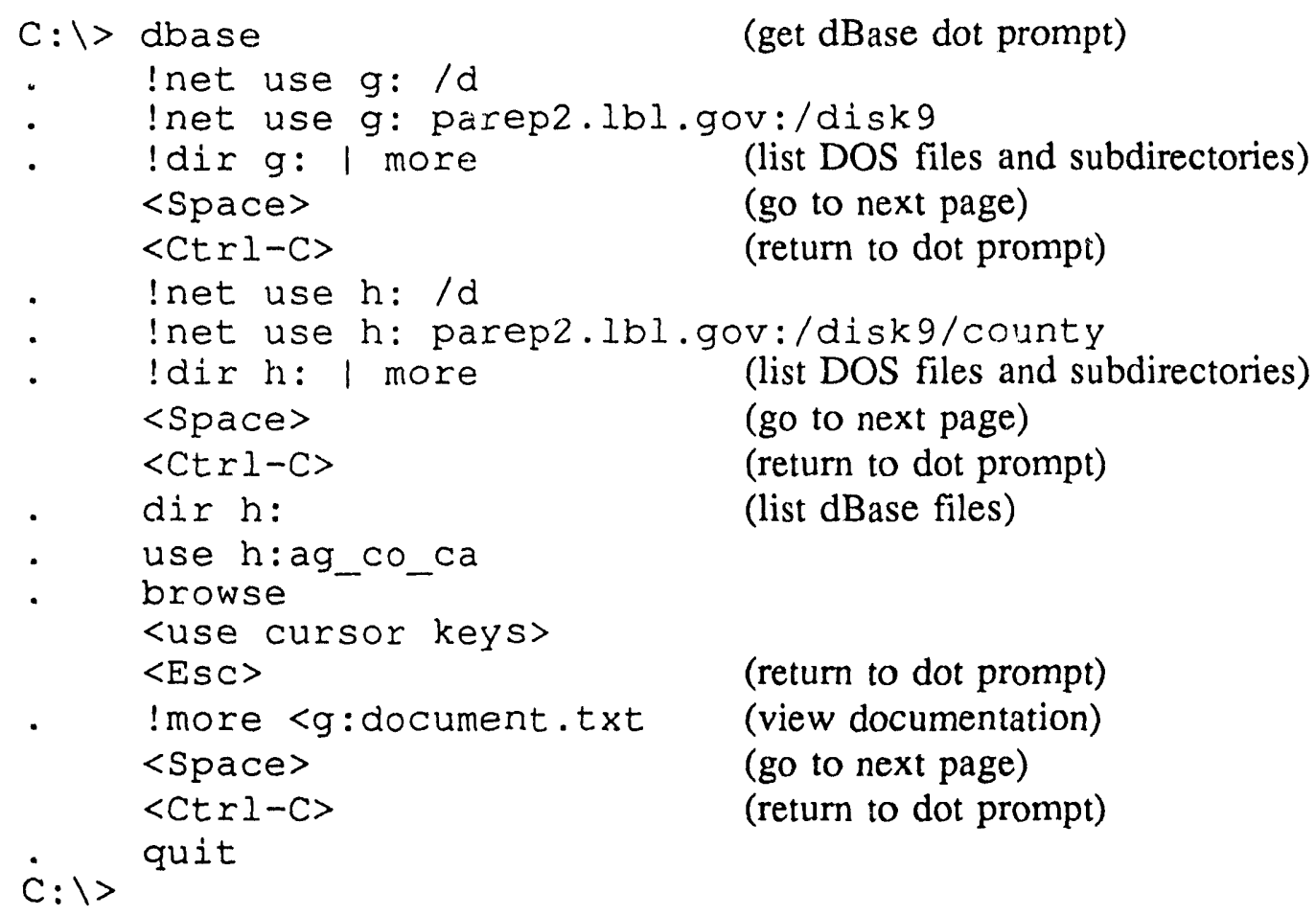




\section{ECONOMIC CENSUSES: RELEASE 1D}

As indicated in Table I, the CD-ROM diskette containing Release 1D of the 1987 Economic Censuses (in dBase format) is:

cedrcd.1bl.gov: /cdroms/disk5

which is currently exported from:

parep2.1bl.gov: /disk5

\section{EXTRACT}

To view the data under PC-NFS with the Census Bureau's program EXTRACT, type the following:

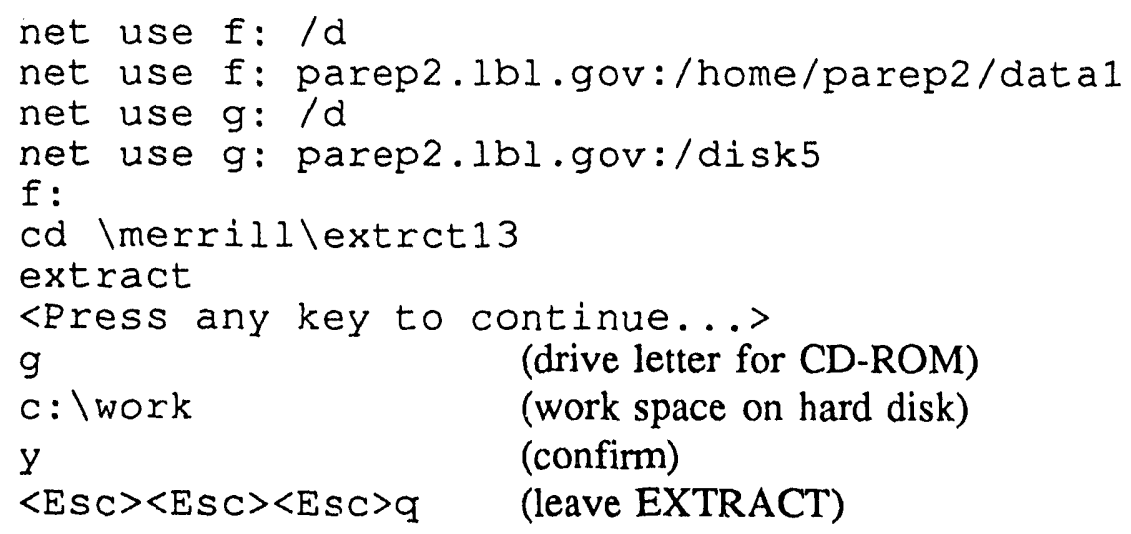




\section{ECONOMIC CENSUSES: RELEASE 2B}

As indicated in Table I, the CD-ROM diskette containing Release 2B of the 1987 Economic Censuses (in dBase format) is:

cedrcd.lbl.gov: /cdromsidisk23

which is currently exported from:

parep2.1bl.gov: /disk23

\section{EXTRACT}

To view the data under PC-NFS with the Census Bureau's program EXTRACT, type the following:

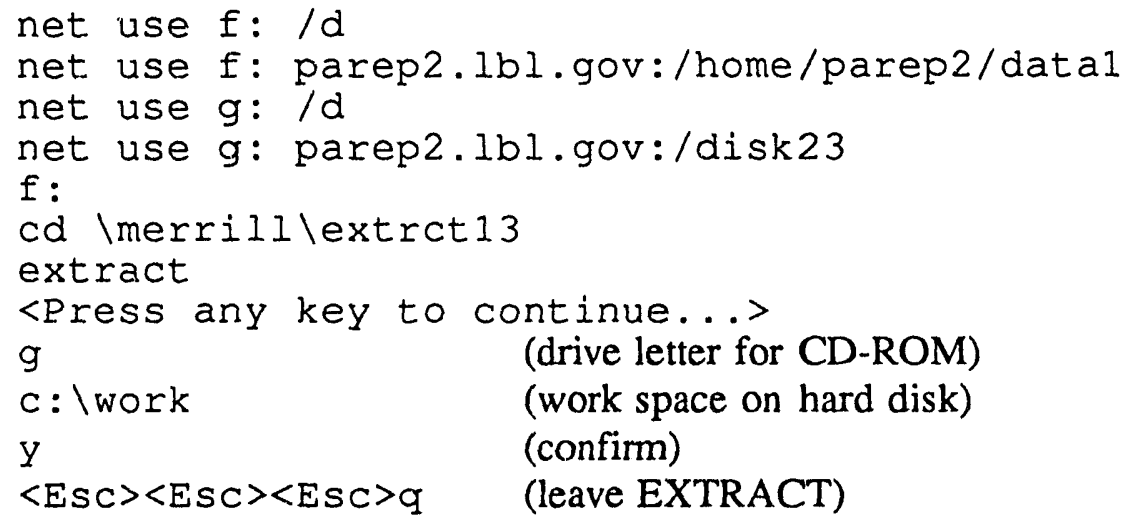




\section{9-90 REGIONAL ECONOMIC INFORMATION SYSTEM (REIS)}

As indicated in Table I, the CD-ROM diskette containing the 1969-90 Regional Economic Information System (REIS) is:

cedrcd.lbl.gov: /caroms/disk92

which is currently exported from:

misty.1bl.gov: /disk92

\section{REIS}

Software for PC-compatible computers is in:

cedrcd.lbl.gov: /datal/merrill/reiscdrm

which is currently exported from:

parep2.lbl.gov: /home/parep2/data1/merrill/reiscdrm

To view the REIS data under PC-NFS, type the following:

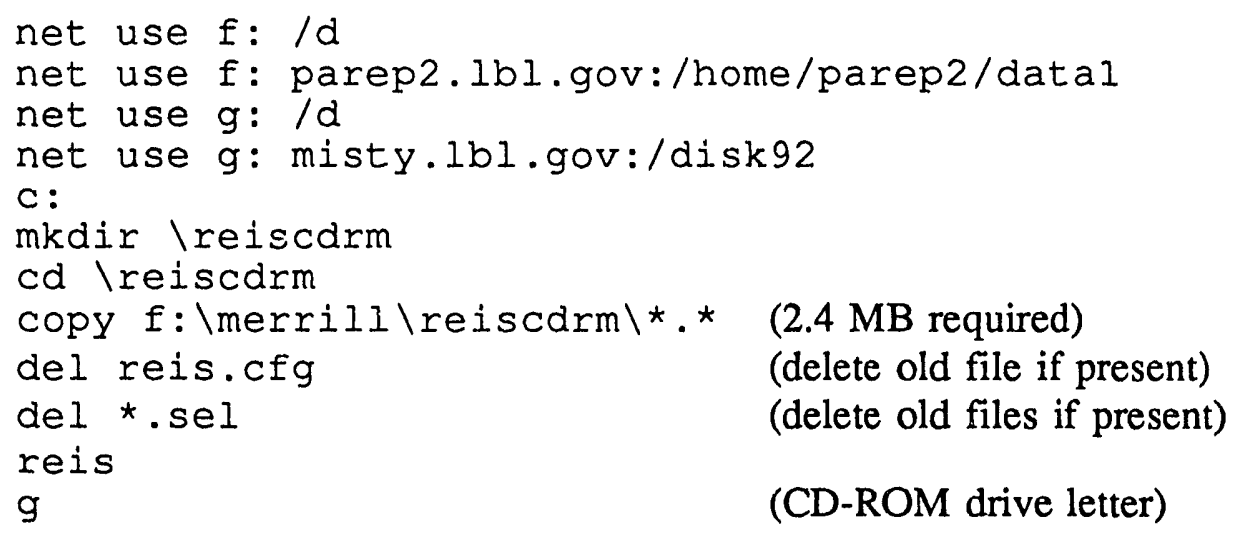

For further information about REIS, contact:

Ken Berkman

Regional Economic Measurement Division (BE-55)

U.S. Department of Commerce

Bureau of Economic Analysis

Washington DC 20230

tel. (202) 523-0552 


\section{8-1991 CURRENT POPULATION SURVEY}

As indicated below, the CD-ROM diskettes containing the 1988-1991 Current Population Survey (CPS) data are in:

cedrcd.lbl.gov: /cdroms/disk8 and /cdroms/disk24

which are currently exported from:

parep2.1bl.gov: /disk8 and /disk24

\section{FILE LOCATIONS}

The locations of Current Population Survey data, by year, are as follows. (years = years of data; date = release date of CD-ROM diskette; location = path name in cedrcd. IbI.gov; no = number of $\mathrm{CD}-\mathrm{ROM}$ diskettes; $\mathrm{MB}=$ size in megabytes.)

\begin{tabular}{||l|l|l|r|r|}
\hline \multicolumn{2}{|c|}{ Table II. 1988-1991 } & Current Population survey \\
\hline years & date & location & no & MB \\
\hline \hline $1988,1989,1990$ & $8 / 91$ & /cdroms/disk8 & 1 & 560 \\
\hline $1989,1990,1991$ & $10 / 91$ & /cdroms/disk24 & 1 & 563 \\
\hline total & & & 2 & 1,123 \\
\hline
\end{tabular}

\section{CHANGES IN FILE NAMES}

Certain file names are changed by PC-NFS. For example, the CD-ROM file name "readme." (lower case, with a trailing period) is translated by PC-NFS into "READM AA" and not "README" . The PC-NFS command is displays both the original CD-ROM file names and the NFS-translated file names. To read the CPS documentation, type:

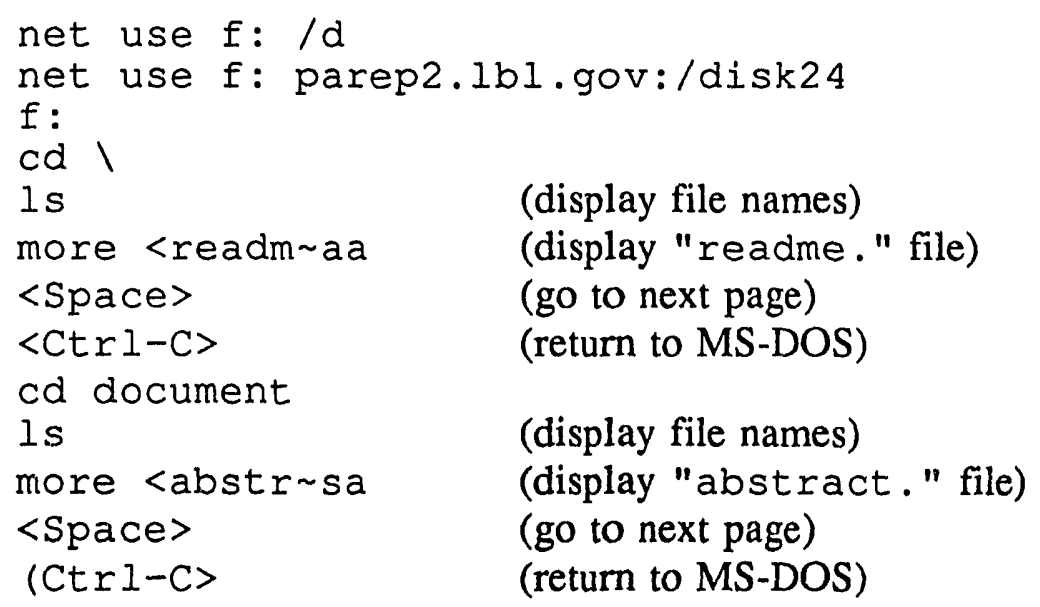

(display file names)

(display "abstract." file)

(go to next page)

(return to MS-DOS) 


\section{CENSUS PL94-171 REDISTRICTING DATA}

As indicated below, the CD-ROM diskettes containing the 1990 Census PL94-171 redistricting data are in:

cedrcd.Ibl.gov: /cdroms/disk10 through/cdroms/disk19

which are currently exported from:

parep2.1bl.gov: /disk10 through /disk19

\section{GO}

To view (for example) California data under PC-NFS with the Census Bureau's GO program, type the following:

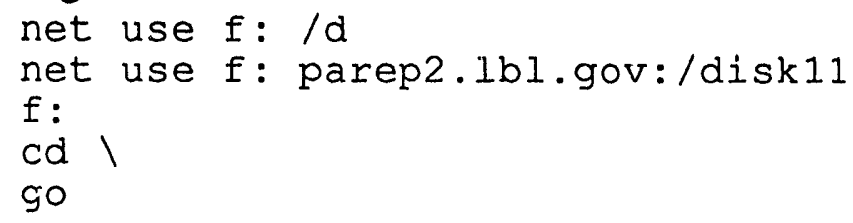




\section{FACTFINDER}

To view (for example) 1990 Census populations of California counties, under PC-NFS with Sammamish Data Systems' proprietary FactFinder program, purchase the PL94-171 extraction module and FactFinder USA, and install in directory $c: \backslash f f$. For further information, contact:

Sammamish DataSystems, Inc.

1813 - 130th Ave NE, Suite 218

Bellevue WA 98005-2240

tel: (206) 867-1485

fax: (206) 861-0184

First, to extract the PL94-171 data from CD-ROM, type:

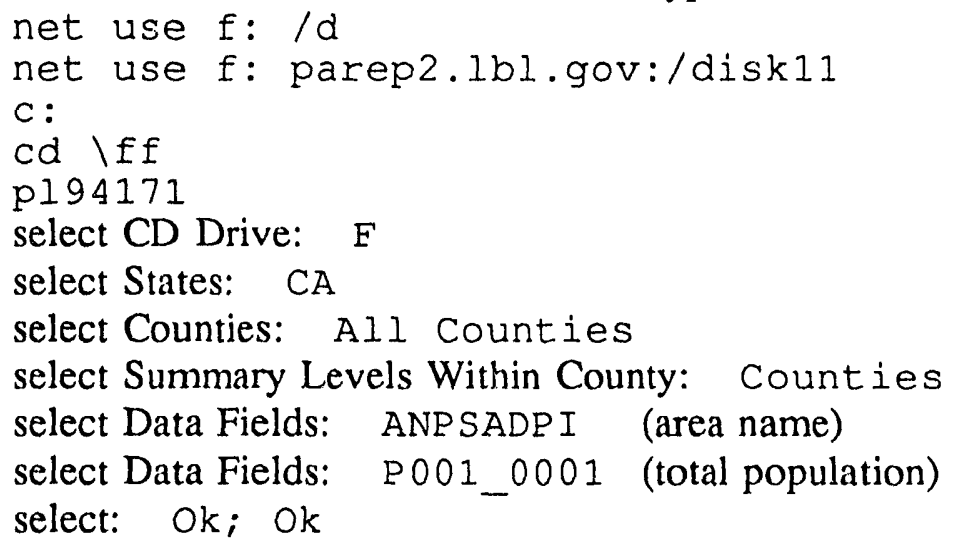

Then, to view the data with FactFinder USA, type:

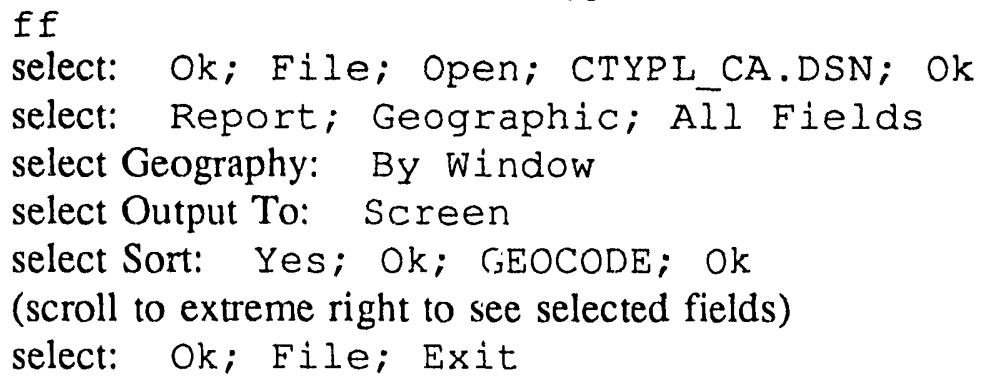




\section{FILE LOCATIONS}

The locations of 1990 Census PL94-171 redistricting data, by state, are as follows. (FIPS = Federal Information Processing System state code; location = path name in cedrcd. $1 \mathrm{bl} . \mathrm{gov} ; \mathrm{MB}=$ size in megabytes; ncy = number of counties.)

\begin{tabular}{|c|c|c|c|c|c|}
\hline state & $a b b r$ & FIPS & location & MB & ncy \\
\hline United States & & & & 5,000 & 3,141 \\
\hline Alabama & $A L$ & 01 & /cdroms/disk13/pl9417al.* & 119 & 67 \\
\hline Alaska & AK & 02 & /cdroms/disk12/p19417ak. * & 14 & 25 \\
\hline Arizona & $\mathrm{AZ}$ & 04 & /cdroms/disk10/p19417az.* & 75 & 15 \\
\hline Arkansas & AK & 05 & /cdroms/disk14/p19417ar.* & 98 & 75 \\
\hline California & $\mathrm{CA}$ & 06 & /cdroms/disk11/p19417ca.* & 357 & 58 \\
\hline Colorado & $\mathrm{CO}$ & 08 & /cdroms/disk12/pl9417co.* & 88 & 63 \\
\hline Connecticut & $\mathrm{CT}$ & 09 & /cdroms/disk15/p19417ct.* & 38 & 8 \\
\hline Delaware & $\mathrm{DE}$ & 10 & /cdroms/disk13/p19417de.* & 11 & 3 \\
\hline $\begin{array}{l}\text { District of } \\
\text { Columbia }\end{array}$ & DC & 11 & /cdroms/disk15/p19417dc. * & 4 & 1 \\
\hline Florida & FL & 12 & /cdroms/disk16/pl9417f1.* & 220 & 67 \\
\hline Georgia & GA & 13 & /cdroms/disk10/p19417ga.* & 142 & 159 \\
\hline Hawaii & $\mathrm{HI}$ & 15 & /cdroms/disk17/p19417hi.* & 11 & 5 \\
\hline Idaho & ID & 16 & /cdroms/disk12/p19417id.* & 41 & 44 \\
\hline Illinois & IL & 17 & /cdroms/disk13/p19417il.* & 246 & 102 \\
\hline Indiana & IN & 18 & /cdroms/disk14/pl9417in.* & 147 & 92 \\
\hline Iowa & IA & 19 & /cdroms/disk18/p19417ia.* & 111 & 99 \\
\hline Kansas & KS & 20 & /cdroms/disk18/pl9417ks. * & 118 & 105 \\
\hline Kentucky & $\mathrm{KY}$ & 21 & /cdroms/disk16/pl9417ky.* & 84 & 120 \\
\hline Louisiana & LA & 22 & /cdroms/disk14/p194171a.* & 109 & 64 \\
\hline Maine & $\mathrm{ME}$ & 23 & /cdroms/disk12/p19417me.* & 36 & 16 \\
\hline Maryland & MD & 24 & /cdroms/disk15/p19417md. * & 59 & 24 \\
\hline Massachusetts & MA & 25 & /cdroms/disk16/p19417ma. * & 79 & 14 \\
\hline Michigan & MI & 26 & /cdroms/disk10/pl9417mi.* & 181 & 83 \\
\hline Minnesota & $\mathrm{MN}$ & 27 & /cdroms/disk18/p19417mn.* & 130 & 87 \\
\hline Mississippi & MS & 28 & /cdroms/disk14/pl9417ms. * & 79 & 82 \\
\hline
\end{tabular}




\begin{tabular}{|c|c|c|c|c|c|}
\hline state & $a b b r$ & FIPS & location & $M B$ & ncy \\
\hline Missouri & MO & 29 & /cdroms/disk17/p19417mo.* & 152 & 115 \\
\hline Montana & $\mathrm{MT}$ & 30 & /cdroms/disk17/pl9417mt. * & 40 & 57 \\
\hline Nebraska & $\mathrm{NE}$ & 31 & /cdroms/disk13/pl9417ne.* & 81 & 93 \\
\hline Nevida & NV & 32 & /cdroms/disk17/p19417nv.* & 25 & 17 \\
\hline New Hampshire & NH & 33 & /cdroms/disk10/p19417nh. * & 23 & 10 \\
\hline New Jersey & $\mathrm{NJ}$ & 34 & /cdroms/disk19/pl9417nj.* & 106 & 21 \\
\hline New Mexico & NM & 35 & /cdroms/disk16/p19417nm.* & 56 & 33 \\
\hline New York & NY & 36 & /cdroms/disk11/p19417ny.* & 231 & 62 \\
\hline North Carolina & NC & 37 & /cdroms/disk15/pl9417nc.* & 158 & 100 \\
\hline North Dakota & ND & 38 & /cdroms/disk10/p19417nd. * & 62 & 53 \\
\hline Ohio & $\mathrm{OH}$ & 39 & /cdroms/disk15/pl9417oh.* & 180 & 88 \\
\hline Oklahoma & OK & 40 & /cdroms/disk13/pl9417ok .* & 116 & 77 \\
\hline Oregon & OR & 41 & /cdroms/disk13/p19417or.* & 69 & 36 \\
\hline Pennsylvania & $\mathrm{PA}$ & 42 & /cdroms/disk18/p19417pa.* & 241 & 67 \\
\hline Rhode Island & RI & 44 & /cdroms/disk15/p19417ri.* & 15 & 5 \\
\hline South Carolina & $\mathrm{SC}$ & 45 & /cdroms/disk12/pl9417sc.* & 99 & 46 \\
\hline South Dakota & $S D$ & 46 & /cdroms/disk14/p19417sd.* & 52 & 66 \\
\hline Tennessee & TN & 47 & /cdroms/disk16/pl9417tn.* & 121 & 95 \\
\hline Texas & $\mathrm{TX}$ & 48 & /cdroms/disk17/p19417tx.* & 345 & 254 \\
\hline Utah & UT & 49 & /cdroms/disk16/p19417ut. * & 37 & 29 \\
\hline Vermont & VT & 50 & /cdroms/disk19/pl9417vt.* & 16 & 14 \\
\hline Virginia & VA & 51 & /cdroms/disk19/p19417va.* & 106 & 136 \\
\hline Washington & WA & 53 & /cdroms/disk12/p19417wa.* & 96 & 39 \\
\hline West Virginia & WV & 54 & /cdroms/disk12/pl9417wv.* & 56 & 55 \\
\hline Wisconsin & WI & 55 & /cdroms/disk10/p19417wi.* & 139 & 72 \\
\hline wyoming & WY & 56 & /cdroms/disk14/p19417wy. * & 37 & 23 \\
\hline
\end{tabular}




\section{TIGER FILES: POSTCENSUS RELEASE}

As indicated below, the CD-ROM diskettes containing the 1990 TIGER files (postcensus release) are in:

cedrcd.1bl.gov: /cdroms/disk25 through/cdroms/disk68

which are currently exported from:

$$
\begin{aligned}
& \text { parep.lbl.gov: /disk25 through/disk } 48 \\
& \text { misty.lbl.gov: /disk49 through/disk68 }
\end{aligned}
$$

The TIGER (Topologically Integrated Geographic Encoding and Referencing) files from the 1990 Census include latitude-longitude coordinates of points which define the boundaries of geographic units such as counties, minor civil divisions, census tracts, block groups, etc. Also included are the coordinates of geographic features such as shorelines, rivers, roads, etc.

\section{FACTFINDER}

To view (for example) census tract boundaries and shorelines in San Francisco county, California, under PC-NFS with Sammamish Data Systems' proprietary FactFinder program, purchase the TIGER/Line File Extraction module and FaciFinder USA, and install in directory $c: \backslash f f \backslash$. For further information, contact:

Sammamish DataSystems, Inc.

1813 - 130th Ave NE, Suite 218

Bellevue WA 98005-2240

tel: (206) 867-1485

fax: (206) 861-0184

First, to extract the TIGER/Line File data from CD-ROM, type:

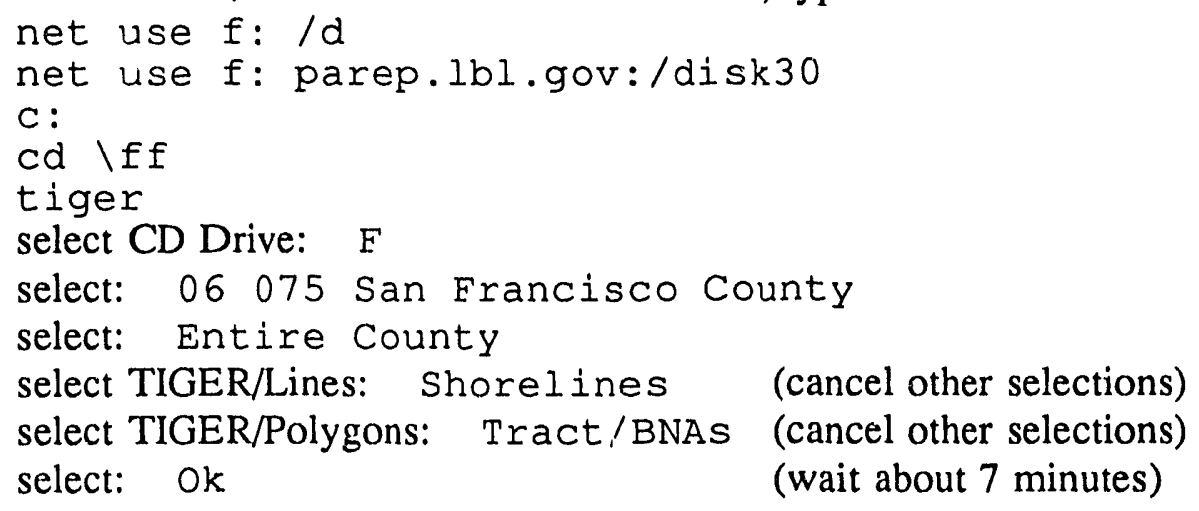

Then, to view the tract and shoreline boundaries with FactFinder USA, type:

ff

select: Ok; File; Open; TGR06075.DSN; Ok

select: File; Exit 


\section{FILE LOCATIONS}

The locations of 1990 postcensus TIGER files, by state, are as follows. (FIPS $=$ Federal Information Processing System state code; call = call number in UC Berkeley Documents Library catalog; location = path name in cedrcd.1bl.gov ; $\mathrm{MB}=$ size in megabytes; ncy $=$ number of counties.) The full call number, where $\langle$ cal 1$\rangle=1$ through 44 , is

H A 2011990 T53 COMPU/D <Call> DOCS

Geographic reference name files are located in /cdroms/disk26/pubgrf $90 . s 01$, etc. Files listed under "MB" do not include geographic reference name files.

\begin{tabular}{|c|c|c|c|c|c|c|}
\hline state / counties & $a b b r$ & FIPS & call & location & $\mathrm{MB}$ & ncy \\
\hline United States & & & & total & 24,000 & 3,141 \\
\hline Alabama & $\mathrm{AL}$ & 01 & 2 & /cdroms/disk26/01 & 552 & 67 \\
\hline Alaska & AK & 02 & 1 & /cdroms/disk25/02 & 109 & 25 \\
\hline Arizona & $\mathrm{Az}$ & 04 & 3 & /cdroms/disk27/04 & 538 & 15 \\
\hline Arkansas & AR & 05 & 4 & /cdroms/disk28/05 & 524 & 75 \\
\hline California & \multirow[t]{4}{*}{$\mathrm{CA}$} & \multirow[t]{4}{*}{06} & & total & 1,405 & 58 \\
\hline $\begin{array}{l}\text { Alameda- } \\
\text { Mono }\end{array}$ & & & 5 & /cdroms/disk29/06 & 613 & 26 \\
\hline $\begin{array}{l}\text { Monterey- } \\
\text { Siskiyou }\end{array}$ & & & 6 & /cdroms/disk30/06 & 624 & 21 \\
\hline Solano-Yuba & & & 7 & /cdroms/disk31/06 & 168 & 11 \\
\hline Colorado & $\mathrm{CO}$ & 08 & 8 & /cdroms/disk32/08 & 498 & 63 \\
\hline Connecticut & $\mathrm{CT}$ & 09 & 9 & /cdroms/disk33/09 & 129 & 8 \\
\hline Delaware & $\mathrm{DE}$ & 10 & 9 & /cdroms/disk33/10 & 39 & 3 \\
\hline $\begin{array}{l}\text { District of } \\
\text { Columbia }\end{array}$ & DC & 11 & 10 & /cdroms/disk34/11 & 7 & 1 \\
\hline Elorida & \multirow[t]{3}{*}{ FL } & \multirow[t]{3}{*}{12} & & total & 836 & 67 \\
\hline $\begin{array}{l}\text { Alachua- } \\
\text { Pasco }\end{array}$ & & & 11 & /cdroms/disk35/12 & 633 & 51 \\
\hline $\begin{array}{l}\text { Pinellas- } \\
\text { Washington }\end{array}$ & & & 12 & /cdroms/disk36/12 & 203 & 16 \\
\hline Georgia & $\mathrm{GA}$ & 13 & 13 & /cdroms/disk37/13 & 645 & 159 \\
\hline Hawaii & $\mathrm{HI}$ & 15 & 1 & /cdroms/disk25/15 & 68 & 5 \\
\hline Idaho & ID & 16 & 12 & /cdroms/disk36/16 & 377 & 44 \\
\hline Illinois & IL & 17 & & total & 732 & 102 \\
\hline
\end{tabular}




\begin{tabular}{|c|c|c|c|c|c|c|}
\hline state / counties & \multirow[t]{3}{*}{$a b b r$} & \multirow[t]{3}{*}{ FIPS } & call & location & MB & ncy. \\
\hline $\begin{array}{l}\text { Adams - } \\
\text { Rock Is Iand }\end{array}$ & & & 14 & /cdroms/disk38/17 & 579 & 81 \\
\hline $\begin{array}{l}\text { St. Clair- } \\
\text { Woodford }\end{array}$ & & & 15 & /cdroms/disk39/17 & 153 & 21 \\
\hline Indiana & IN & 18 & 15 & /cdroms/disk39/18 & 449 & 92 \\
\hline Iowa & IA & 19 & 16 & /cdroms/disk $40 / 19$ & 429 & 99 \\
\hline Kansas & KS & 20 & 17 & /cdroms/disk $41 / 20$ & 587 & 105 \\
\hline Kentucky & KY & 21 & 18 & /cdroms/disk $42 / 21$ & 456 & 120 \\
\hline Louisisana & LA & 22 & 19 & /cdroms/disk $43 / 22$ & 484 & 64 \\
\hline Maine & $\mathrm{ME}$ & 23 & 20 & /cdroms/disk $44 / 23$ & 187 & 16 \\
\hline Maryland & MD & 24 & 21 & /cdroms/disk $45 / 24$ & 211 & 24 \\
\hline Massachusetts & $\mathrm{MA}$ & 25 & 20 & /cdroms/disk $44 / 25$ & 248 & 14 \\
\hline Michigan & MI & 26 & 22 & /cdroms/disk $46 / 26$ & 600 & 83 \\
\hline Minnesota & $\mathrm{MN}$ & 27 & 23 & /cdroms/disk $47 / 27$ & 556 & 87 \\
\hline Mississippi & MS & 28 & 24 & /cdroms/disk $48 / 28$ & 465 & 82 \\
\hline Missouri & \multirow[t]{3}{*}{ Mo } & \multirow[t]{3}{*}{29} & & total & 683 & 115 \\
\hline Adair-Texas & & & 25 & /cdroms/disk49/29 & 639 & 107 \\
\hline Vernon-Wright & & & 17 & /cdroms/disk41/29 & 44 & 8 \\
\hline Montana & $\mathrm{MT}$ & 30 & 26 & /cdroms/disk50/30 & 593 & 57 \\
\hline Nebraska & $\mathrm{NE}$ & 31 & 27 & /cdroms/disk51/31 & 400 & 93 \\
\hline Nevada & NV & 32 & 7 & /cdroms/disk31/32 & 359 & 17 \\
\hline New Hampshire & $\mathrm{NH}$ & 33 & 20 & /cdroms/disk $44 / 33$ & 96 & 10 \\
\hline New Jersey & $\mathrm{NJ}$ & 34 & 9 & /cdroms/disk33/34 & 263 & 21 \\
\hline New Mexico & NM & 35 & 28 & /cdroms/disk52/35 & 531 & 33 \\
\hline New York & NY & 36 & 29 & /cdroms/disk53/36 & 644 & 62 \\
\hline North Carolina & \multirow[t]{3}{*}{ NC } & \multirow[t]{3}{*}{37} & & total & 706 & 100 \\
\hline $\begin{array}{l}\text { Alamance- } \\
\text { Stokes }\end{array}$ & & & 30 & /cdroms/disk54/37 & 605 & 85 \\
\hline $\begin{array}{l}\text { Surry- } \\
\text { Yancey }\end{array}$ & & & 31 & /cdroms/disk55/37 & 101 & 15 \\
\hline North Dakota & ND & 38 & 32 & /cdroms/disk56/38 & 325 & 53 \\
\hline Ohio & $\mathrm{OH}$ & 39 & 33 & /cdroms/disk57/39 & 633 & 88 \\
\hline Oklahoma & OK & 40 & 34 & /cdroms/disk58/40 & 550 & 77 \\
\hline
\end{tabular}




\begin{tabular}{|c|c|c|c|c|c|c|}
\hline state / counties & $a b b r$ & EIPS & call & location & $\mathrm{MB}$ & ncy \\
\hline Oregon & OR & 41 & 35 & /cdroms/disk59/41 & 590 & 36 \\
\hline Pennsylvania & \multirow[t]{3}{*}{$\mathrm{PA}$} & \multirow[t]{3}{*}{42} & & total & 778 & 67 \\
\hline $\begin{array}{l}\text { Adams - } \\
\text { Schuy } 1 k 121\end{array}$ & & & 36 & /cdroms/disk60/42 & 643 & 54 \\
\hline Snyder-York & & & 9 & /cdroms/disk33/42 & 135 & 13 \\
\hline Rhode Island & RI & 44 & 9 & /cdroms/disk33/44 & 43 & 5 \\
\hline South Carolina & SC & 45 & 31 & /cdroms/disk55/45 & 407 & 46 \\
\hline South Dakota & SD & 46 & 37 & /cdroms/disk61/46 & 369 & 66 \\
\hline Tennessee & $\mathrm{TN}$ & 47 & 38 & /cdroms/disk $62 / 47$ & 548 & 95 \\
\hline Texas & \multirow[t]{5}{*}{$\mathrm{TX}$} & \multirow[t]{5}{*}{48} & & total & 1,929 & 254 \\
\hline $\begin{array}{l}\text { Anderson- } \\
\text { Fayette }\end{array}$ & & & 39 & /cdroms/disk63/48 & 614 & 75 \\
\hline $\begin{array}{l}\text { Fisher- } \\
\text { McCulloch }\end{array}$ & & & 40 & /cdroms/disk64/48 & 582 & 79 \\
\hline $\begin{array}{l}\text { McLennan- } \\
\text { Wheeler }\end{array}$ & & & 41 & /cdroms/disk65/48 & 642 & 88 \\
\hline $\begin{array}{l}\text { Wichita- } \\
\text { Zavala }\end{array}$ & & & 34 & /cdroms/disk58/48 & 91 & 12 \\
\hline Utah & UT & 49 & 32 & /cdroms/disk56/49 & 301 & 29 \\
\hline Vermont & VT & 50 & 20 & /cdroms/disk44/50 & 69 & 14 \\
\hline Virginia & VA & 51 & 10 & /cdroms/disk34/51 & 539 & 136 \\
\hline Washington & WA & 53 & 42 & /cdroms/disk66/53 & 549 & 39 \\
\hline West Virginia & wV & 54 & 21 & /cdroms/d1sk45/54 & 297 & 55 \\
\hline Wisconsin & WI & 55 & 43 & /cdroms/disk67/55 & 529 & 72 \\
\hline Wyoming & WY & 56 & 44 & /cdroms/disk68/56 & 403 & 23 \\
\hline outlying areas & & & & total & 235 & 111 \\
\hline American Samoa & AS & 60 & 1 & /cdroms/disk25/60 & 4 & 5 \\
\hline $\begin{array}{l}\text { Federated States } \\
\text { of Micronesia }\end{array}$ & & 64 & 1 & /cdroms/disk25/64 & 7 & 4 \\
\hline Guam & GU & 66 & 1 & /cdroms/disk25/66 & 9 & 1 \\
\hline Marshall Islands & & 68 & 1 & /cdroms/disk25/68 & 5 & 33 \\
\hline $\begin{array}{l}\text { Northern Mariana } \\
\text { Islands }\end{array}$ & CM & 69 & 1 & /cdroms/disk25/69 & 4 & 4 \\
\hline Palau & & 70 & 1 & /cdroms/disk25/70 & 5 & 16 \\
\hline Puerto Rico & PR & 72 & 1 & /cdroms/disk25/72 & 193 & 78 \\
\hline
\end{tabular}




\begin{tabular}{||l|l|l|l|l|r|r||}
\hline \multicolumn{7}{|c|}{ Table IV. 1990 TIGER files: postcensus release } \\
\hline \hline state / counties & abbr & FIPS & call & location & MB & ncy \\
\hline $\begin{array}{l}\text { U.S. Minor } \\
\text { Outiying Islands } \\
\text { (Midway Islands) }\end{array}$ & & 74 & 1 & /cdroms/disk25/74 & 0.02 & 1 \\
\hline $\begin{array}{l}\text { Virgin Islands } \\
\text { of the United } \\
\text { States }\end{array}$ & VI & 78 & 1 & /cdroms/disk25/78 & 8 & 3 \\
\hline
\end{tabular}




\section{CENSUS: SUMMARY TAPE FILE 1A (STF1A)}

As indicated below, a sample CD-ROM diskette containing 1990 Census Summary Tape File 1A (STF1A) data for California is:

$$
\text { cedrcd.lbl.gov: /cdroms/disk22 }
$$

which is currently exported from:

$$
\text { parep2.1bl.gov: /disk22 }
$$

\section{FILE LOCATIONS}

The locations of 1990 Census Summary Tape File 1A files, by state, are as follows. (FIPS = Federal Information Processing System state code; location = path name in cedrcd.1bl.gov ; $\mathrm{MB}=$ size in megabytes; $\mathrm{ncy}=$ number of counties.)

\begin{tabular}{|l|l|l|l|l|r|}
\hline \multicolumn{5}{|c|}{ Table V. 1990 Census: Summary Tape File 1A (STF1A) } \\
\hline state & abbr & FIPS & location. & MB & ncy \\
\hline California & CA & 06 & /cdroms/disk22/*Ca.* & 656 & 58 \\
\hline
\end{tabular}

\section{GO}

To view (for example) California data under PC-NFS with the Census Bureau's GO program, type the following:

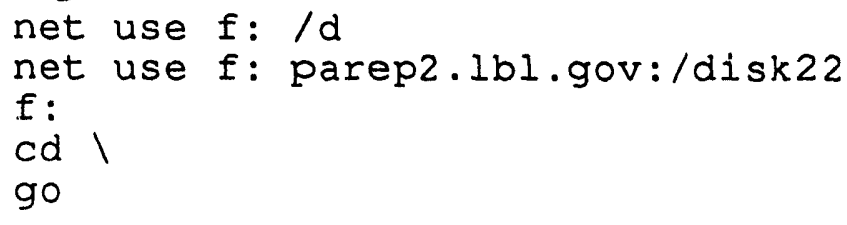




\section{FACTFINDER}

To view (for example) 1990 Census populations of California counties, under PC-NFS with Sammamish Data Systems' proprietary FactFinder program, purchase the STF1A extraction module and FactFinder USA, and install in directory $c: \backslash f f$. For further information, contact:

Sammamish DataSystems, Inc.

1813 - 130th Ave NE, Suite 218

Bellevue WA $98005-2240$

tel: (206) 867-1485

fax: (206) 861-0184

First, to extract the STF1A data from CD-ROM, type:

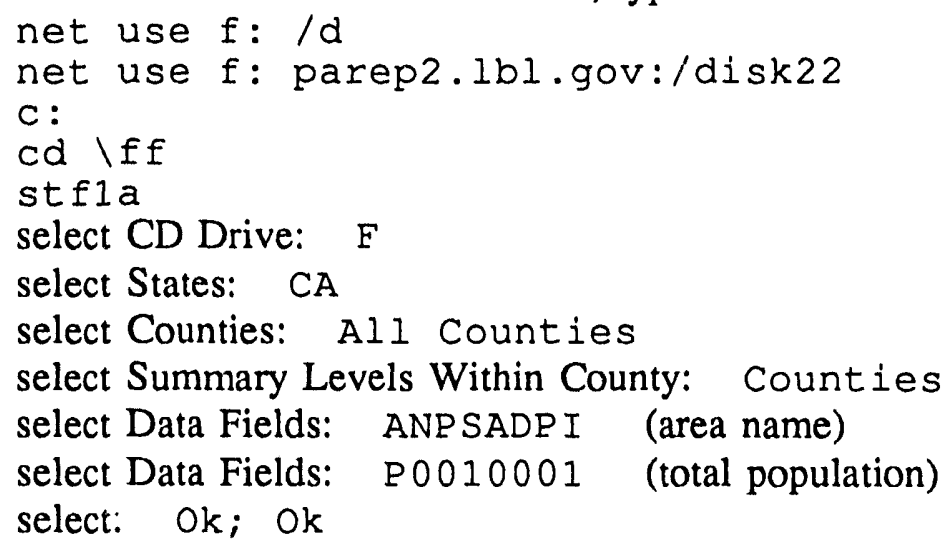

Then, to view the data with FactFinder USA, type:

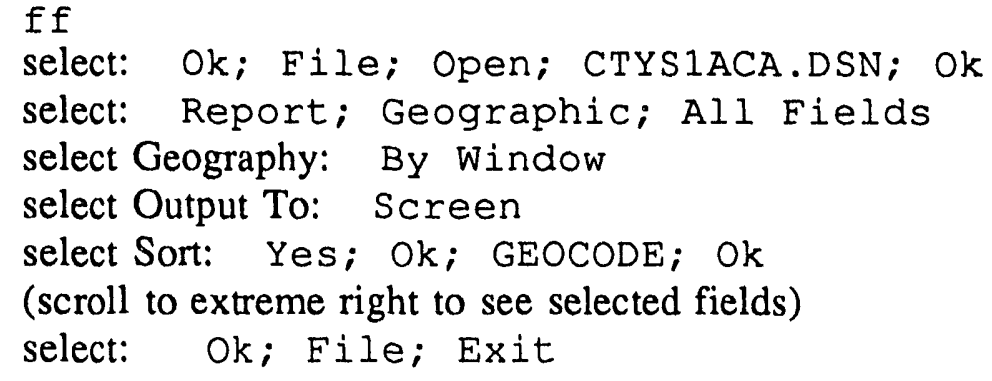




\section{CENSUS: SUMMARY TAPE FILE 1C (U.S. SUMMARY)}

As indicated in Table I, the CD-ROM diskette containing U.S. summary data from 1990 Census Summary Tape File 1C (STF1C) is: cedrcd.lbl.gov: /cdroms/disk89

which is currently exported fium: parep.lbl.gov: /disk89

GO

To view STF1C data under PC-NFS with the Census Bureau's GO program, type the following:

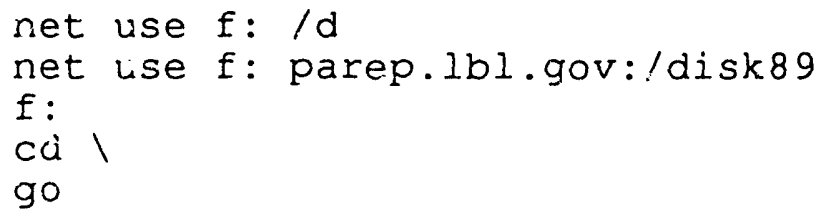




\section{FACTFINDER}

To view (for example) 1990 Census populations of U.S. states under PC-NFS with Sammamish Data Systems' proprietary FactFinder program, purchase the STF1C extraction module and FactFinder USA, and install in directory $c: \backslash f f$. For further information, contact:

Sammamish DataSystems, Inc.

1813 - 130th Ave NE, Suite 218

Bellevue WA $98005-2240$

tel: (206) $867-1485$

fax: (206) 861-0184

First, to extract the STF1C data from CD-ROM, type:

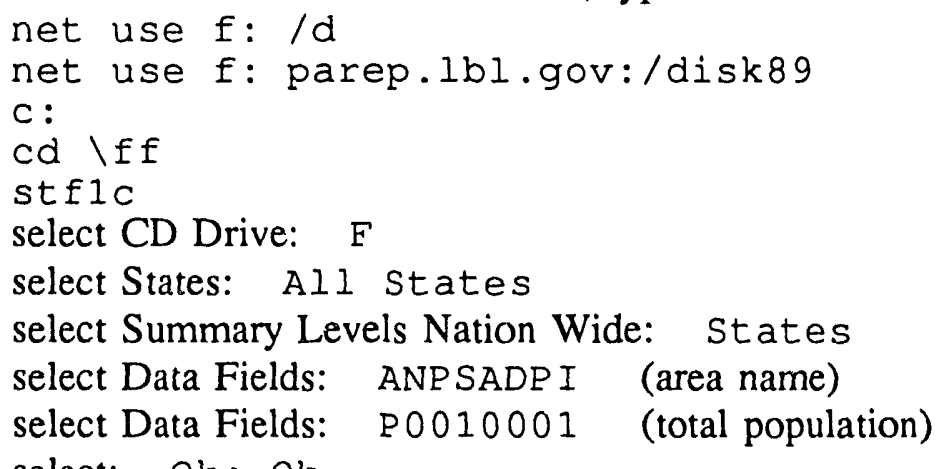

Then, to view the data with FactFinder USA, type:

ff

select: Ok; File; Open; STASICUS.DSN; Ok

select: Report; Geographic; All Fields

select Geography: By Window

select Output To: Screen

select Sort: Yes; Ok; GEOCODE; Ok

(scroll to citreme right to see selected fields)

select: Ok; File; Exit 


\section{CENSUS: SUMMARY TAPE FILE 3A (STF3A)}

As indicated below, CD-ROM diskettes containing 1990 Census Summary Tape File 3A (STF3A) data for six counties in the Los Angeles area are: cedrcd.lbl.gov: /cdroms/disk69 and /cdroms/disk70

which are currently exported from:

misty.1bl.gov: /disk69 and /disk70

\section{FILE LOCATIONS}

The locations of STF3A files, by state and county, are as follows. (FIPS = Federal Information Processing System state code; date = release date of CD-ROM diskette; location = path name in cedrcd.1bl.gov ; $\mathrm{MB}=$ size in megabytes; $\mathrm{ncy}=$ number of counties.)

The Los Angeles county data (on /cdroms/disk69) are from a prototype CD-ROM diskette produced in May 1992. This diskette contains minor errors and will be replaced later.

\begin{tabular}{|c|c|c|c|c|c|}
\hline state / counties & FIPS & date & location & MB & ncy \\
\hline California (partial) & 06 & & & 1,258 & 6 \\
\hline Los Angeles & 037 & $5 / 92$ & $\begin{array}{l}\text { /cdroms } \\
\text { disk69 }\end{array}$ & 639 & 1 \\
\hline $\begin{array}{l}\text { Orange, Riverside, San } \\
\text { Bernardino, San Diego, } \\
\text { Ventura }\end{array}$ & $\begin{array}{l}059,065, \\
071,073, \\
111\end{array}$ & $10 / 92$ & $\begin{array}{l}\text { /cdroms/ } \\
\text { disk } 70\end{array}$ & 619 & 5 \\
\hline
\end{tabular}

GO

To view (for example) Orange county data under PC-NFS with the Census Bureau's GO program, type the following:

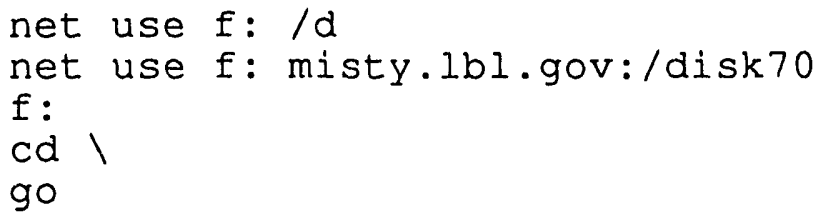




\section{FACTFINDER}

To view (for example) 1990 Census populations of five California counties under PC-NFS with Sammamish Data Systems' proprietary FactFinder program, purchase the STF3A extraction module and FactFinder USA, and install in directory $c: \backslash f f$. For further information, contact:

Sammamish DataSystems, Inc.

1813 - 130th Ave NE, Suite 218

Bellevue WA 98005-2240

tel: (206) 867-1485

fax: (206) 861-0184

First, to extract the STF3A data from CD-ROM, type:

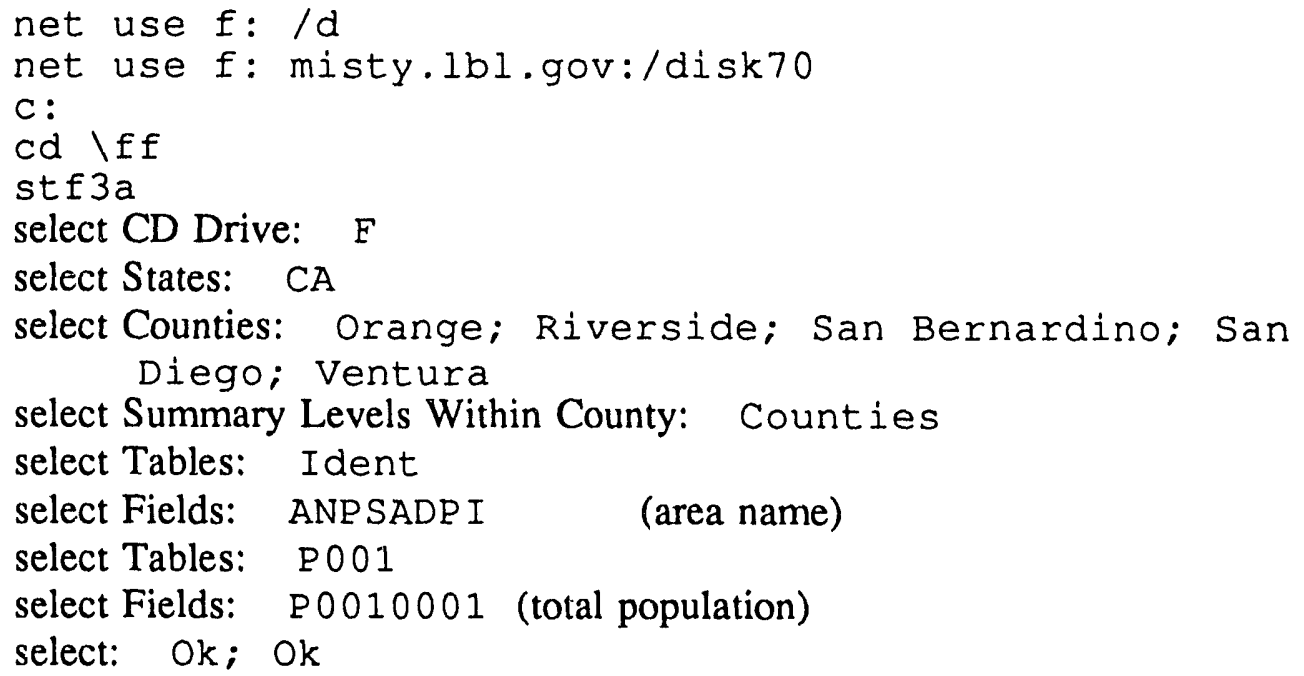

Then, to view the data with FactFinder USA, type:

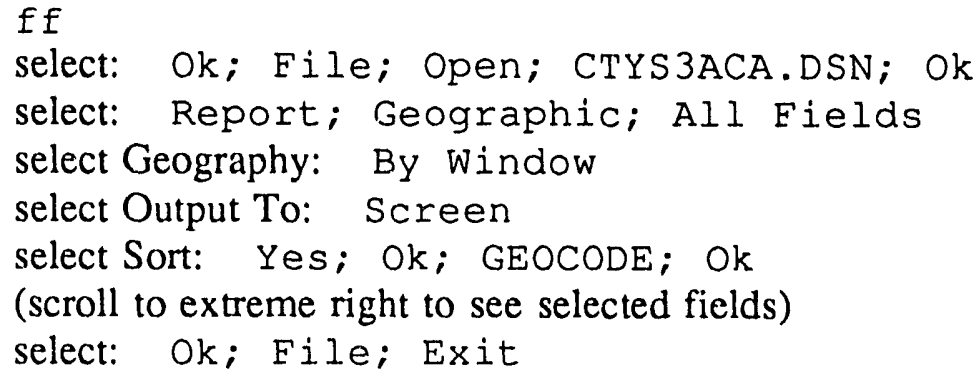




\section{USA COUNTY STATISTICS}

As indicated in Table I, a CD-ROM diskette containing USA County Statistics data is: cedrcd.lbl.gov: /cdroms/disk90

which is currently exported from:

parep.Ibl.gov: /disk90

GO

To view the USA County Statistics data under PC-NFS with the Census Bureau's GO program, type the following:

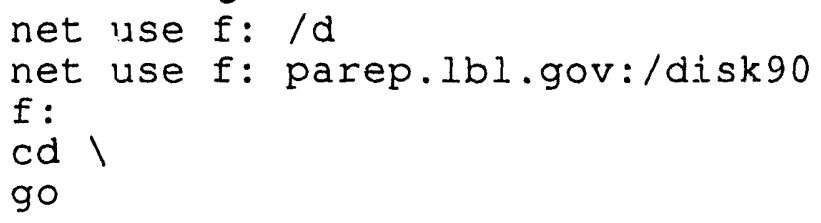

\section{CHANGES IN FILE NAMES}

Certain file names are changed by PC-NFS. For example, the CD-ROM file name "readme." (lower case, with a trailing period) is translated by PC-NFS into "READM AA" and not "README" . The PC-NFS command is displays both the original CD-ROM file names and the NFS-translated file names. To read the USA County Statistics documentation, type:

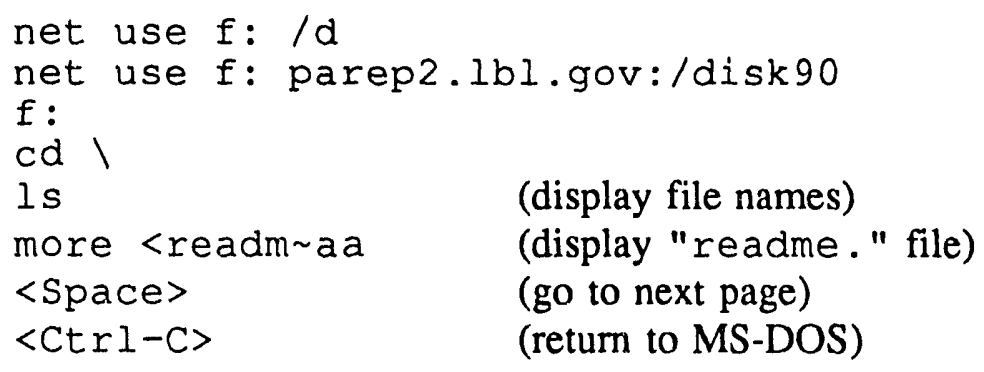




\section{DIGITAL CHART OF THE WORLD}

The Digital Chart of the World (DCW) was developed by the United States Defense Mapping Agency (DMA) with the cooperation of Australia, Canada, and the United Kingdom.

As indicated below, the CD-ROM diskettes containing DCW are:

cedrcd.lbl.gov: /cdroms/disk85 through /cdroms/disk8 8

which are presently exported from

parep.lbl.gov: /disk85 through /disk88

\section{FILE LOCATIONS}

Locations of DCW on CD-ROM are as follows: (location = path name in cedrcd. $1 \mathrm{bl}$.gov ; $\mathrm{MB}=$ size in megabytes.)

\begin{tabular}{|l|l|r|}
\hline \multicolumn{2}{|c|}{ Table VII. Digital Chart of the World } \\
\hline \hline continents & location & MB \\
\hline North America & /cdroms/disk85 & 388 \\
\hline Europe/Northern Asia & /cdroms/disk86 & 365 \\
\hline South America/Africa/Antarctica & /cdroms/disk87 & 406 \\
\hline Southern Asia/Australia & /cdroms/disk88 & 428 \\
\hline \hline & total & 1,587 \\
\hline
\end{tabular}




\section{VPFVIEW}

A public domain MS-DOS program VPFVIEW (including source code) for use with DCW is available from the location cedrcd.1bl.gov: /datal/merrill/dcw10

which is presently exported from parep2.lbl.gov: /home/parep2/datal/merrill/dcw10

To install VPFVIEW in a local directory c: \vpfview type the following. Seven megabytes of disk space are required.

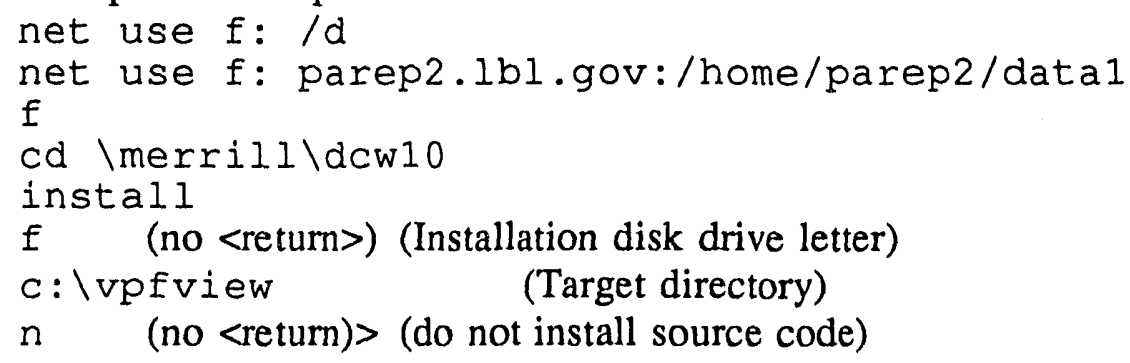

VPFVIEW, DCW and PC-NFS are mutually incompatible, due to CD-ROM file names in DCW of the type "dht." and "lat." (lower case with trailing period) which are translated by PC-NFS into "DHT TE" and "LAT UE" rather than "DHT" and "LAT" . In order to use VPFVIEW with DCW under PC-NFS, one must do one of the following:

(a) copy DCW to a local disk and change file names (for example) to "DHT" and "LAT" ;

(b) modify VPFVIEW;

(c) modify PC-NFS.

Option (a) has been used at LBL. A modified portion of DCW; namely, the worldwide summary BROWSE database; is in the location cedrcd.lbl.gov: /data1/merrill/dcw

which is presently exported from parep2.lbl.gov: /home/parep2/datal/merrill/dcw

To run VPFVIEW on the (modified) BROWSE database, type:

net use $f: / d$

net use f: parep2.1bl.gov:/home/parep2/datal

c :

cd Ivpfview vpfview

$f: \backslash$ merrill \dcw (mouse click on BROWSE )

(the path to a VPF database)

(select a Library) 


\section{NATIONAL HEALTH INTERVIEW SURVEY (NHIS)}

As indicated in Table I, a CD-ROM diskette containing data from the 1992 National Health Interview Survey is:

cedrcd.lbl.gov: /cdroms/disk71

which is currently exported from:

misty.lbl.gov: /disk71

\section{SETS}

To view the NHIS data with the Statistical Export and Tabulation System (SETS), make sure that your file c: \config.sys specifies at least 2000 bytes of environment space and at least 30 files, for example:

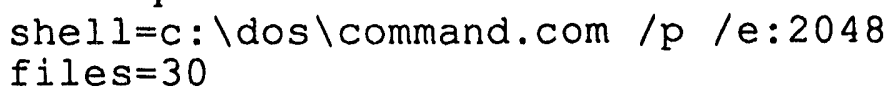

Also make sure your hard disk $c$ : has at least five megabytes of free space. Install SETS on your hard disk as follows (it will be installed in directory c: \nhis1988):

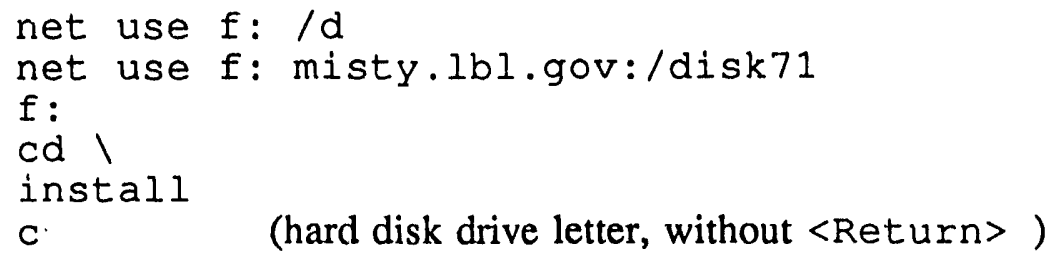

To view the data with SETS, type:

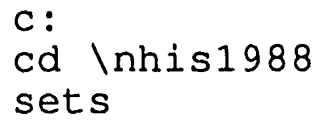

c:

cd \nhis 1988

sets

Online assistance is available. To obtain SETS software and documentation and further information, contact:

National Center for Health Statistics

Scientific and Technical Information Branch

6525 Belcrest Road

Hyattsville MD 20782

tel: (301) 436-8500 


\section{ANONYMOUS FTP}

Computer files described in this document are available via anonymous FTP from

Internet node:

cedrcd. lbl.gov

$(128.3 .196 .16)$

this document (PostScript):

/data1/merril1/docs/cedr/cdrom3.ps data on CD-ROM:

/cdroms

Due to the size of the files, access via NFS is preferable to FTP. Please contact Deane Merrill (dwmerrillelbl.gov) if you wish to make use of the data. 


\section{NFS SERVERS}

Currently, documentation and software are NFS-exported from the following location:

LBL Information and Computing Sciences Division:

parep2.lb1.gov 128.3.196.16 /home/parep2/data1

CD-ROM diskettes are exported from the following locations:

\begin{tabular}{|c|c|c|c|c|c|}
\hline & $\begin{array}{l}\text { parep2.1bl.gov } \\
\text { parep.ibl.gov } \\
\text { misty.1bl.gov } \\
\text { parep.1bl.gov } \\
\text { misty.1bl.gov } \\
\text { parep2.1bi.gov } \\
\text { bigsur.lbl.gov }\end{array}$ & $\begin{array}{l}128 \cdot 3 \cdot 196.16 \\
128 \cdot 3 \cdot 254.131 \\
128 \cdot 3 \cdot 140.150 \\
128 \cdot 3.254 .131 \\
128 \cdot 3.140 .150 \\
128.3 .196 .16 \\
128.3 .140 .31\end{array}$ & $\begin{array}{l}\text { /disk1 } \\
\text { /disk25 } \\
\text { /disk49 } \\
\text { /disk85 } \\
\text { /disk91 } \\
\text { /disk115 } \\
\text { /disk121 }\end{array}$ & $\begin{array}{l}\text {-/disk24 } \\
\text {-/disk48 } \\
\text {-/disk72 } \\
\text {-/disk90 } \\
\text {-/disk96 } \\
\text {-/disk120 } \\
\text {-/disk138 }\end{array}$ & $\begin{array}{r}\left(\begin{array}{rl}24 \\
( & 24 \\
( & 24\end{array}\right) \\
\left(\begin{array}{r}6 \\
(\end{array}\right) \\
\left(\begin{array}{rl}6 \\
(\end{array}\right) \\
(18)\end{array}$ \\
\hline & $\begin{array}{l}\text { csm29.berkeley.edu } \\
\text { csm29.berkeley.edu }\end{array}$ & $\begin{array}{l}128.32 .165 .47 \\
128.32 .165 .47\end{array}$ & $\begin{array}{l}\text { /disk73 } \\
\text { /disk97 }\end{array}$ & $\begin{array}{l}\text {-/disk84 } \\
\text {-/disk114 } \\
\text { otal: }\end{array}$ & $\begin{array}{l}\left(\begin{array}{l}12) \\
(18) \\
(138)\end{array}\right) \\
(13)\end{array}$ \\
\hline
\end{tabular}

The 138 exported locations include the 72 diskettes listed in Table I, and 66 locations reserved for future use:

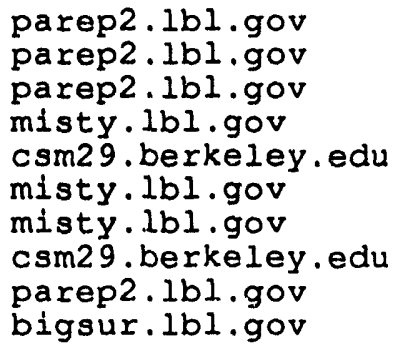

$128 \cdot 3 \cdot 196 \cdot 16$
$128 \cdot 3 \cdot 196 \cdot 16$
$128 \cdot 3 \cdot 196 \cdot 16$
$128 \cdot 3 \cdot 140 \cdot 150$
$128 \cdot 32 \cdot 165 \cdot 47$
$128 \cdot 3 \cdot 140.150$
$128 \cdot 3 \cdot 140.150$
$128 \cdot 32 \cdot 165 \cdot 47$
$128 \cdot 3 \cdot 196 \cdot 16$
$128 \cdot 3 \cdot 140 \cdot 31$

\begin{tabular}{|c|c|}
\hline $\begin{array}{l}\text { /disk2 } \\
\text { /disk6 } \\
\text { /disk20 } \\
\text { /disk72 } \\
\text { /disk73 } \\
\text { /disk91 } \\
\text { /disk93 } \\
\text { /disk97 } \\
\text { /disk115 } \\
\text { /disk121 }\end{array}$ & $\begin{array}{l}\text {-/disk3 } \\
\text {-/disk7 } \\
\text {-/disk21 } \\
\text {-/disk84 } \\
\text {-/disk96 } \\
\text {-/disk114 } \\
\text {-/disk120 } \\
\text {-/disk138 } \\
\text {-tal: }\end{array}$ \\
\hline
\end{tabular}




\section{NFS CLIENTS}

Please contact Deane Merrill (dwmerrillelbl.gov) if you wish to have the CD-ROMs exported to your PC or work station. Please provide (following the examples below): name of your organization and department; internet address, IP address, and hardware type of your computer; name, phone, and electronic address of your system administrator. Access may be restricted in the future if costs become excessive.

Currently, data on CD-ROM are exported to the following NFS clients. File locations as seen by the user are determined by the NFS client system administrator.

( $\mathrm{M}=$ mounted by NFS client)

( $E=$ exported, not yet mounted)

At this time, cedrcd.1bl.gov is synonymous with parep2.1bl.gov. That equivalence is subject to change.

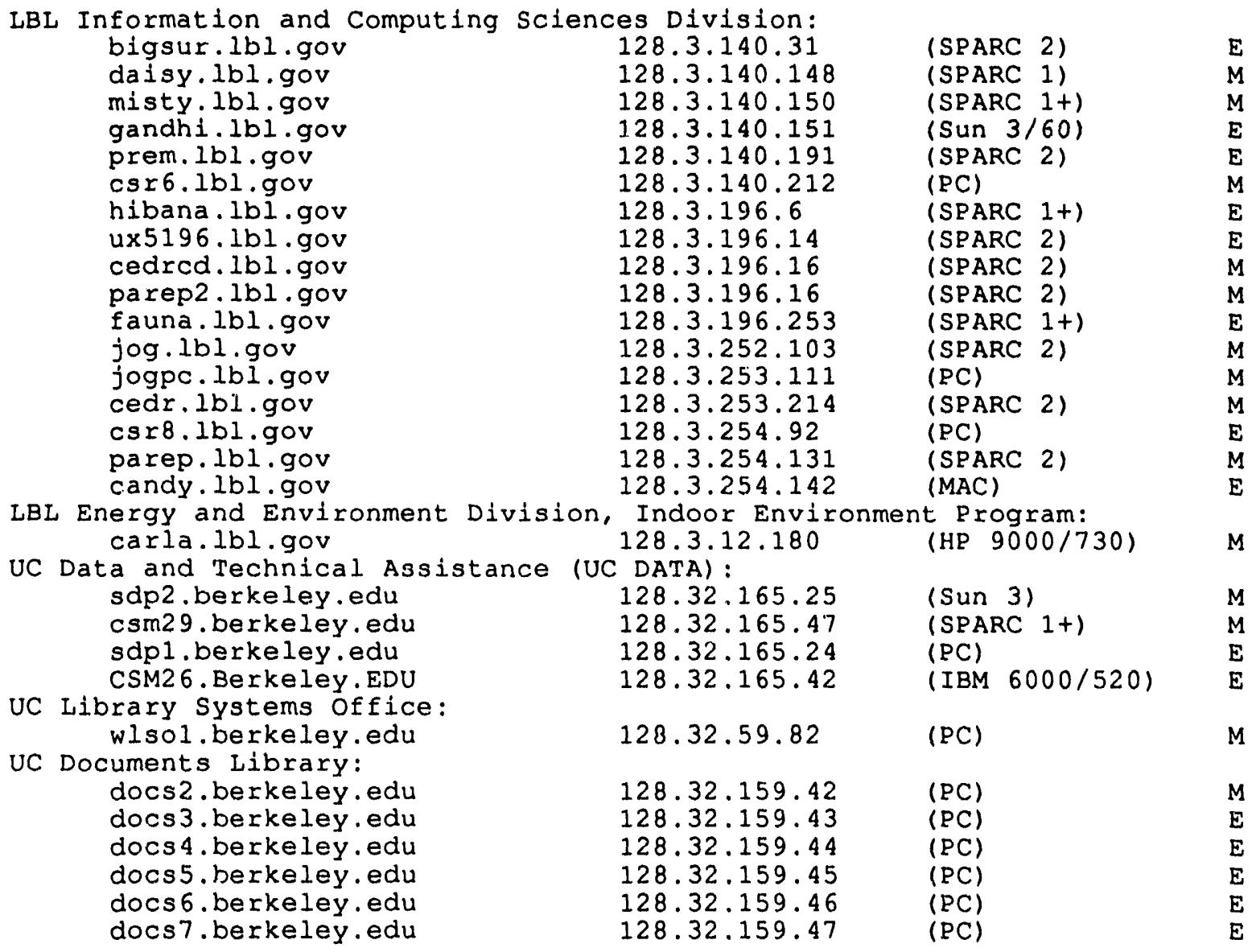


UC Berkeley Regional Planning and Center for Environmental Design:

redwood.ced. berkeley.edu

sibley.ced.berkeley.edu

lurd-j.ced.berkeley.edu

dean 1 . ced.berkeley.edu

sonoma. ced.berkeley. edu

128.32 .151 .61

128.32 .151 .66

128.32 .151 .233

128.32 .181 .203

128.32 .181 .106

(SPARC)

(SPARC)

(PC)

(PC)

(SPARC)

$M$

UC Berkeley Demography Department: lotka.qal. berkeley .edu

128.32 .163 .162 king .qal berkeley.edu

UC Berkeley School of Public Health: psi.berkeley.edu

128.32 .203 .44

(SPARC)

Lawrence Livermore National Laboratory Systems Development Engineering: burrito.11nl.gov

128.115 .32 .2

(SPARC 2)

UC Davis Computing Services:

lystra.ucdavis.edu

$128.120 .132 .103 \quad$ (PC)

E 


\section{SYSTEM ADMINISTRATORS}

The following system administrators should be contacted for technical information about specific NFS servers or NFS clients:

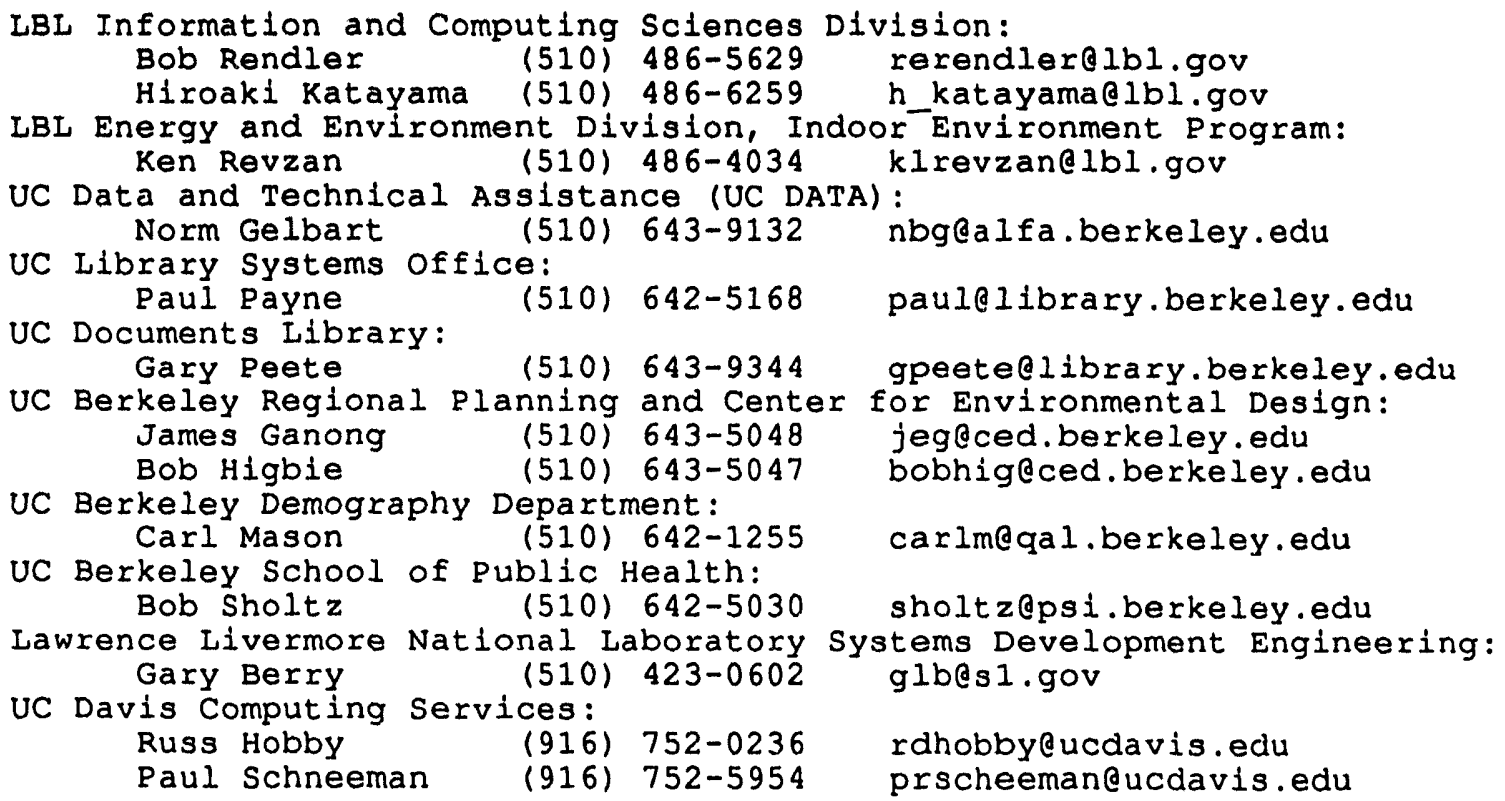




\section{MOUNTING CD-ROMS ON A SUN SPARC STATION}

To NFS-mount the CD-ROM diskettes on a Sun SPARC station, you must first make an export request as described under "NFS Clients."

After the CD-ROM diskettes are exported to your work station, log in as root, append the 139 lines below to the system file /etc/fstab, and type

mount -av

Then $\log$ in as an ordinary user and try to read a few of the files. Make sure to test at least one diskette from each NFS server, for example

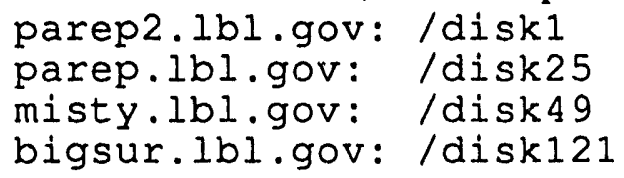

The server csm29. berkeley. edu, for which 30 slots are reserved, is not functioning at present.

NOTE: The 66 comment lines preceded by \# indicate locations reserved for future use. Mounting these locations prematurely by removing the \# will cause no harm (and will eliminate future effort), but their contents are not documented and are subject to change without notice.

\begin{tabular}{|c|c|}
\hline 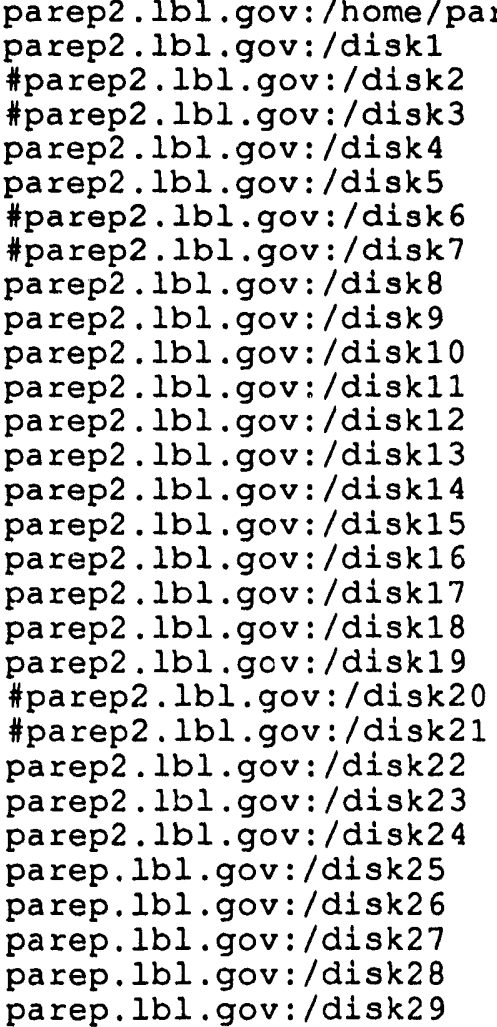 & $\begin{array}{l}\text { /cdroms/disk1 } \\
\text { /cdroms/disk2 } \\
\text { /cdroms/disk3 } \\
\text { /cdroms/disk4 } \\
\text { /cdroms/disk5 } \\
\text { /cdroms/disk6 } \\
\text { /cdroms/disk7 } \\
\text { /cdroms/disk8 } \\
\text { /cdroms/disk9 } \\
\text { /cdroms/disk10 } \\
\text { /cdroms/disk11 } \\
\text { /cdroms/disk12 } \\
\text { /cdroms/disk13 } \\
\text { /cdroms/disk14 } \\
\text { /cdroms/disk15 } \\
\text { /cdroms/disk16 } \\
\text { /cdroms/disk17 } \\
\text { /cdroms/disk18 } \\
\text { /cdroms/disk19 } \\
\text { /cdroms/disk20 } \\
\text { /cdroms/disk21 } \\
\text { /cdroms/disk22 } \\
\text { /cdroms/disk23 } \\
\text { /cdroms/disk24 } \\
\text { /cdroms/disk25 } \\
\text { /cdroms/disk26 } \\
\text { /cdroms/disk27 } \\
\text { /cdroms/disk28 } \\
\text { /cdroms/disk29 }\end{array}$ \\
\hline
\end{tabular}

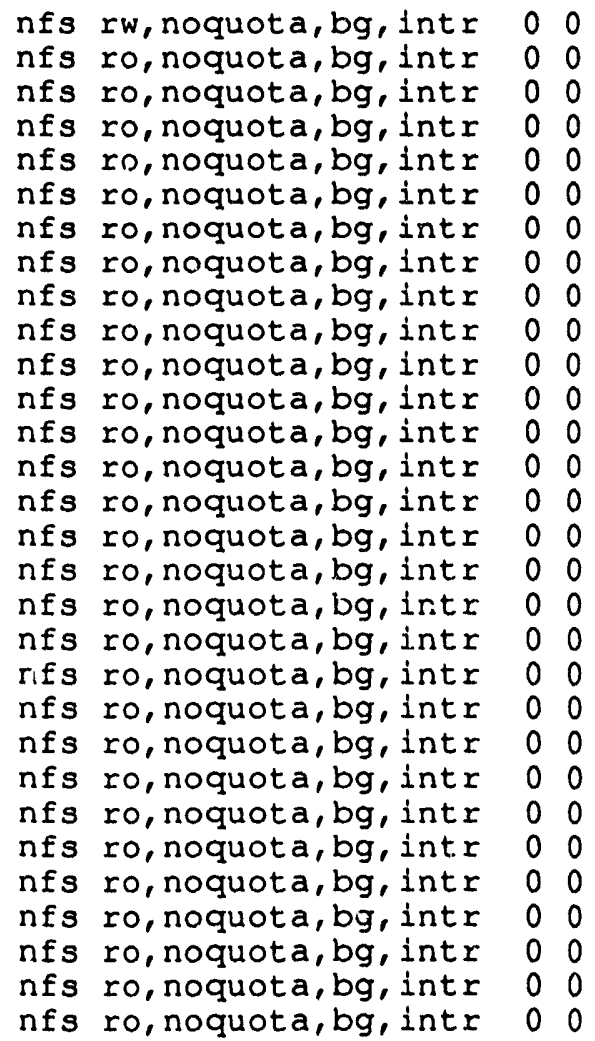


parep.lbl.gov:/disk30 parep.lbi.gov:/disk31 parep.1bl.gov:/disk32 parep. Ibl.gov:/disk33 parep.Ibl.gov:/disk34 parep.lbl.gov:/disk35 parep.1bl.gov:/disk.36 parep.lbl.gov:/disk37 parep.lbl.gov:/disk38 parep.lbl.gov:/disk39 parep.lbl.gov:/disk40 parep.lbl.gov: /disk41 parep.lbl.gov:/disk42 parep.lbl.gov:/disk43 parep. Ibl.gov:/disk44 parep.ibl.gov:/disk4t parep. Ibl.gov:/disk 46 parep.lbl.gov:/disk47 parep.1bl.gov:/disk48 misty.lbl.gov:/disk49 misty. Ibl.gov: /disk50 misty.1bl.gov:/disk51 misty.1bl.gov:/disk52 misty.lbl.gov:/disk53 misty. Ibl.gov:/disk54 misty.lol.gov:/disk55 misty. 1bl.gov:/disk56 misty.1bl.gov:/disk57 misty. Ibl.gov:/disk58 misty.1bl.gov:/disk59 misty.1bl.gov:/disk60 misty. lbl.gov:/disk61 misty.1bl.gov:/disk.62 misty. $1 \mathrm{bl}$.gov:/disk63 misty.1bl.gov: /disk64 misty.1bl.gov:/disk65 misty.lbl.gov:/disk66 xisty.1bl.gov:/disk67 misty.lbl.gov:/disk68 misty.1bl.gov:/disk69 misty.1bl.gov:/disk70 misty.1bl.gov:/disk.71 *misty. lbl.gov:/disk72 *csm29.berkeley.edu: / disk 73 * csm29.berkeley.edu:/disk74 * csm29.berkeley.edu: /disk75 * csm29. berkeley.edu: /disk76 * csm29.berkeley.edu:/disk 77 * csm29.berkeley. edu: /disk 78 * csm29.berkeley.edu:/disk79 * csm29. berkeley.edu:/disk80 * csm29.berkeley.edu: /disk 81 * csm29.berkeley.edu: / di =kô2 2 \# csm29. berkeley. edu:/disk83 * csm29.berkeley.edu:/disk 84 parep. lbl.gov:/disk85 parep. 1bl.gov:'disk86 parep. lbl.gov: / disk 87 parep.1bl.gov: /disk83 parep. lbl.gov:/disk89 parep. ibl.gov:/disk90 *misty. 1bl.gov:/disk91 /cdroms/disk 30 /cdroms/disk 31 /cdroms/disk 32 /cdroms/disk 33 /cdroms/disk34 /cdroms/disk35 /cdroms/disk36 /cdroms/disk 37 /caroms/disk 38 icdroms/disk39 /cdroms/disk 40 /cdroms/disk 41 /cdroms/disk 42 /cdroms/disk43 /cdroms/disk 44 /cdroms/disk45 /cdroms/disk 46 /cdroms/disk 47 /cdroms/disk49 /cdroms/disk49 /cdroms/disk 50 /cdroms/disk 51 /cdroms/disk 52 /cdroms/disk 53 /cdroms/disk 54 /cdroms/disk55 /cdroms/disk56 /cdroms/disk 57 /caroms/disk 58 /cdroms/disk 59 /cdroms/disk 60 /cdroms/disk 61 /cdroms/disk 62 /cdroms/disk 63 icdroms/disk 64 /cdroms/disk 65 /cdroms/disk 66 /cdroms/disk 67 /cdroms/disk 68 /cdroms/disk 69 /cdroms/disk 70 /cdroms/disk 7 /cdroms/disk 72 /cdroms/disk73 /cdroms/disk 74 /cdroms/disk 75 /cdroms/disk 76 /cdroms/disk 77 /cdroms/aisk 78 /cdroms/disk79 /cdroms/disk 80 /cdroms / disk 81 /cdroms/disk 82 /cdroms/ciisk 83 /cdroms/disk 84 /cdroms, disk 85 /cdroms/disk 86 /cdroms/disk 87 /cdroms/disk 88 /cdroms/disk89 /cdroms/disk 90 /cdroms/disk91 nfs ro, noquota, bg, intr nfs ro, noquota, bg, intr nfs ro, noquota, bg, int $r$ nfs ro, noquota, bg, intr nfs ro, noquot $a, b g$, intr nfs ro, noquota, bg, intr nfs ro, noquota, bg, intr nfs ro, noquota, bg, intr nfs ro, noquota, bg, intr nfs ro, noquota, bg, intr nfs ro, noquota, bg, intr nfs ro, noquota, bg, intr nfs $r o$, noquota, bg, intr nfs $x o$, noquota, bg, intr nfs ro, noquota, bg, intr nfs $r c$, noquota, bg, intr nfs ro, noquota, bg, intr ifs ro, noquota, bg, intr nfs ro, noquota, bg, intr nfs ro, noquota, bg, intr nfs ro, noquota, bg, intr nfs ro, noquota, bg, intr. nfs ro, noquota, bg, int $r$ nfs ro, noquota, bg, intr nfs ro, noquota, bg, intr $n f s$ ro, noquota, bg, intr nfs ro, noquota, bg, intr nfs ro, noquota, bg, intr $n \in s$ ro, noquota, bg, intr nfs ro, noquote, bg, intr nfs ro, noquota, $b g$, int $r$ nfs ro, noquota, bg, int $r$ nfs ro, noquota, bg, intr nfs ro, noquota, bg, intr nfs ro, noquota, bg, intr nfs ro, noquota, bg, int $r$ nfs ro, noquota, bg, intr nfs ro, noquota, bg, intr nfs ro, noquota, bg, intr $n f s$ ro, noquota, bg, intr $n f s$ ro, noquota, bg, intr nfs ro, noquota, bg, intr nfs ro, noquota, bg, intr rfs ro, noquota, bg, intr Ifs ro, noquota, bg, intr ifs ro, noquota, bg, intr rfs ro, noquota, bg, intr Ifs ro, noquota, bg, intr Ifs ro, noquota, bg, intr rfs ro, noquota, bg, intr rfs ro, noquota, bg, int $r$ rfs ro, noquota, bg, intr ifs ro, noquota, bg, intr $r f s$ ro, noquota, bg, intr ifs ro, noquota, bg, intr nfs ro, noquota, bg, intr nfs ro, noquota, bg, intr nfs ro, noquota, bg, intr nfs ro, noquota, bg, intr nfs ro, noquota, bg, intr nfs ro, noquota, bg, intr nfs ro, noquota, bg, intr
00 $0 \quad 0$ $\begin{array}{ll}0 & 0\end{array}$ 00 $\begin{array}{ll}0 & 0\end{array}$ $\begin{array}{ll}0 & 0\end{array}$ $\begin{array}{lll}0 & 0 \\ 0 & 0 & 0\end{array}$ 0 $\begin{array}{ll}0 & 0\end{array}$ $\begin{array}{lll}0 & 0\end{array}$ 0 $0 \quad 0$ 0 $0 \quad 0$ $\begin{array}{ll}0 & 0\end{array}$ $\begin{array}{ll}0 & 0\end{array}$ 00 00 00 00 00 $\begin{array}{ll}0 & 0\end{array}$ 00 00 00 00 00 00 $\begin{array}{ll}0 & 0\end{array}$ 00 00 00 00 00 00 00 00 00

00

00

00

00

00 00

00 00

00 00 00 $\begin{array}{ll}0 & 0\end{array}$ 00 00 00 00 00 00 00 00 00

00 00 00 
mist 1 . 1bl.gov:/disk92

\#misty.lbl.gov:/disk93

\#misty. lbl.gov:/disk94

\#misty.lbl.gov:/uisk95

\#misty.lbl.gov:/disk96

\#csm29.berkeley.edu:/disk 97

\# csm29.berkeley.edu:/disk 98

\# csm29.berkeley.edu:/disk99

\# csm29.berkeley.edu : /disk100

\#csm29.berkeley.edu:/disk101

\#csm29.berkeley.edu:/disk102

\#csm29.berkeley.edu:/disk103

\#csm29 . berkeley. edu : /disk104

\#csm29.berkeley.edu:/disk105

\#csm129.berkeley.edu:/disk106

\# ssm29.berkeley.edu:/disk107

\#csm29.berkeley.edu:/disk108

\#csm29.berkeley.edu:/disk10s

\#csm29.berkeley.edu:/disk110

\#csm29.berkeley.edu:/disk111

\#csm29.berkeley.edu:/disk112

\# csm29.berkeley.edu:/disk113

\#csm29.berkeley.edu:/disk114

\#parep2 . lbl.gov:/disk115

\#parep2 . Ibl.gov:/disk116

* parep2 . 1bl.gov:/disk117

\#parep2. Ibl.gov:/disk118

*parep2 . 1bl.gov:/disk119

\#parep2. lbl.gov:/disk120

\#bigsur. Ibl.gov:/disk121

\#bigsur. Ibl.gov:/disk 122

\#bigsur. Ibl.gov:/disk 123

\#bigsur. lbl.gov:/disk 124

\#bigsur. lbl.gov:/disk 125

\#bigsur. Ibl.gov:/disk126

\#higsur. 1bl.gov:/disk127

\#bigsur. 1bl.gov:/disk128

\#bigsur. 1 bl.gov:/disk129

*bigsur. lbl.gov:/disk130

\#bigsur. lbl.gov:/disk131

\#bigsur. lbl.gov:/disk132

\#bigsur. Ibl.gov:/disk133

\#bigsur. Ibl.gov:/disk134

\#bigsur.lbl.gov:/disk135

\#bigsur. Ibl.gov:/disk136

\#bigsur. 1bl.gov:/disk137

\#bigsur. lbl.gov:/aisk 138 /cdroms/disk 92

/cdroms/disk93

/cdroms/disk94

/cdroms/disk95

/cdroms/disk96

/cdroms/disk97

/cdroms/disk98

/cdroms/disk 99

/cdroms/disk100

/cdroms/disk101

/cdroms/disk 102

/cdroms/disk103

/cdroms/disk104

/cdroms/disk105

/cdroms/disk106

/cdroms/disk107

/cdroms/disk108

/cdroms/disk109

/cdroms/disk 110

/cdroms/disk111

/cdroms/disk112

/cdroms/disk113

/cdroms/disk114

icdroms/disk115

/cdroms/disk116

/cdroms/disk 117

/cdroms/disk118

/cdroms/disk119

/cdroms/disk120

/cdroms/disk121

/cdroms/disk 122

/cdroms/disk123

/cdroms/disk124

/cdroms/disk125

/cdroms/disk126

/cdroms/disk127

/cdroms/disk128

/cdroms/disk129

/cdroms/disk130

/cdroms/disk131

/cdroms/disk 132

/cdroms/disk133

/cdroms/disk134

/cdroms/disk135

/cdroms/disk136

/cdroms/disk 137

/cdroms/disk138 nfs ro, noquota, bg, intr nfs ro, noquota, bg, intr nfs ro, noquota, bg, intr nfs ro, noquota, bg, int $r$ nfs ro, noquota, bg, intr rfs ro, noquota, bg, intr rfs ro, noquota, bg, int $r$ rfs ro, noquota, bg, intr ifs ro, noquota, bg, intr rfs ro, noquot $a, b g$, int $x$

rfs ro, noquota, bg, int $r$

rfs ro, noquota, bg, int $r$

rfs ro, noquota, bg, intr

rfs ro, noquota, bg, intr

$r f s$ ro, noquota, bg, int $r$

rfs ro, noquota, bg, intr

rfs ro, noquota, bg, intr

rfs ro, noquota, bg, intr

rfs ro, noquota, bg, intr

ifs ro, noquota, bg, intr

rfs ro, noquota, bg, int $x$

rfs ro, noquota, bg, intr

rfs ro, noquota, bg, intr

Ifs ro, noquota, bg, intr

rfs ro, noquota, bg, intr

rfs ro, noquota, bg, intr

rfs ro, noquota, bg, int $r$

rfs ro, noquota, bg, intr

rfs ro, noquota, bg, intr

rfs ro, noquota, bg, intr

rfs ro, noquota, bg, intr

Ifs ro, noquota, bg, intr

Ifs ro, noquota, bg, intr

ifs ro, noquota, bg, int $r$

Ifs ro, noquota, bg, int $r$

rfs ro, noquota, bg, int $r$

rfs ro, noquota, bg, intr

rfs ro, noquota, bg, intr

rfs ro, noquota, bg, intr

rfs ro, noquota, bg, intr

rfs ro, noquota, bg, intr

Ifs ro, noquota, bg, intr

rfs ro, noquota, bg, intr

ifs ro, noquota, bg, intr

rfs ro, noquota, bg, intr

Ifs ro, noquota, bg, intr

rfs ro, noquota, bg, intr $\begin{array}{ll}0 & 0 \\ 0 & 0\end{array}$

$0 \quad 0$

$\begin{array}{ll}0 & 0 \\ 0 & 0\end{array}$

$\begin{array}{lll}0 & 0\end{array}$

0

$0 \begin{array}{ll}0 & 0\end{array}$

$0 \begin{array}{ll}0 & 0 \\ 0 & 0\end{array}$

$\begin{array}{ll}0 & 0\end{array}$

00

00

$0 \quad 0$

00

0

00

00

00

00

00

0

00

00

0

$0 \quad 0$

00

00

00

00

00

$0 \quad 0$

$\begin{array}{ll}0 & 0\end{array}$

00

$\begin{array}{ll}0 & 0\end{array}$

$\begin{array}{ll}0 & 0\end{array}$

$\begin{array}{ll}0 & 0\end{array}$

00

0

$\begin{array}{ll}0 & 0 \\ 0 & 0\end{array}$

$\begin{array}{ll}0 & 0\end{array}$

0

$\begin{array}{ll}0 & 0\end{array}$

$\begin{array}{ll}0 & 0\end{array}$

00

00

$\begin{array}{ll}0 & 0\end{array}$

$\begin{array}{ll}0 & 0\end{array}$

$\begin{array}{ll}0 & 0\end{array}$

00 


\section{MOUNTING CD-ROMS ON A PC UNDER PC-NFS}

To mount the CD-ROM diskettes on a PC-compatible computer under PC-NFS, you must first make an export request as described under "NFS Clients."

On your PC, after installation of PC-NFS, the location of the PC-NFS program files (for example $c: \backslash n f s)$ is specified in a path statement in the file $c: \backslash a u t o e x e c . b a t$. The following lines should be appended to the file $c: \backslash n f s \backslash$ hosts

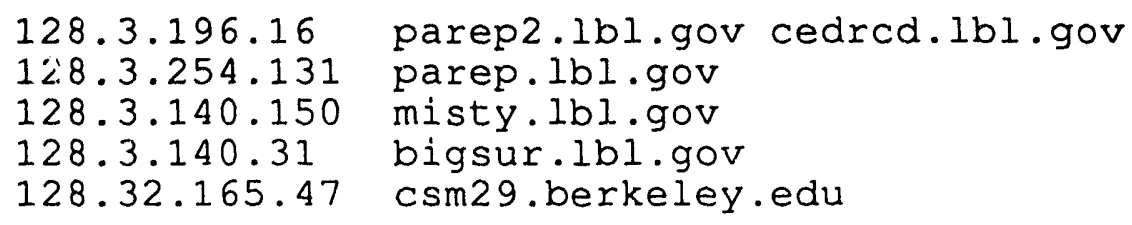

After the CD-ROM directories have been exported to your PC, you should mount (for example) at least one diskette from each server by typing the following lines, where $f:$, $g:, h:, i:$ are unused DOS drive letters:

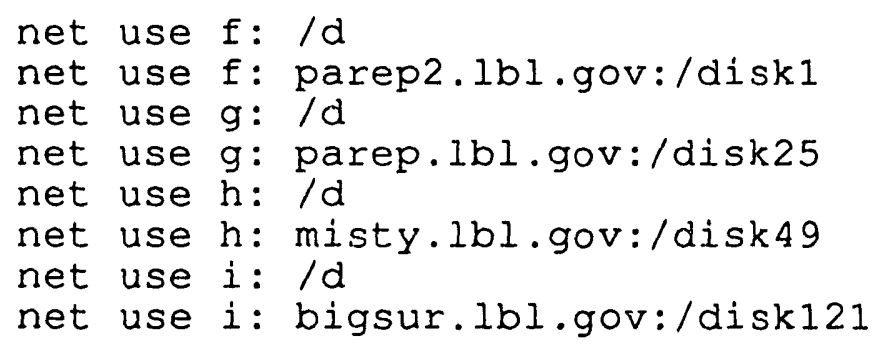

The server csm29.berkeley.edu, for which 30 slots are reserved, is not functioning at present. 


\section{ACKNOWLEDGMENTS}

The author wishes to acknowledge assistance and support provided by Harvard Holmes, Fred Gey, Carl Quong, Bob Rendler, Hiro Katayama, Maggie Morley, Norm Gelbart, Gary Peete, Mari Miller, Andrea Sevetson and Paul Payne.

Network File System (NFS) and PC-NFS are trade marks of Sun Microsystems, Inc. dBase is a trade mark of Borland International. FactFinder and FactFinder USA are trade marks of Sammamish DataSystems. DOS and Microsoft Word are trade marks of Microsoft Corporation. WordPerfect is a trade mark of WordPerfect Corporation. Macintosh and AppleTalk are trade names of Apple Computer, Inc. Mention of a particular product in this report does not constitute endorsement of that product.

This work was supported by the Director, Office of Epidemiology and Health Surveillance, Office of Health, Office of Environment, Safety and Health, U.S. Department of Energy under Contract No. DE-AC03-76SF00098. 


\section{FURTHER INFORMATION}

For further information, contact:

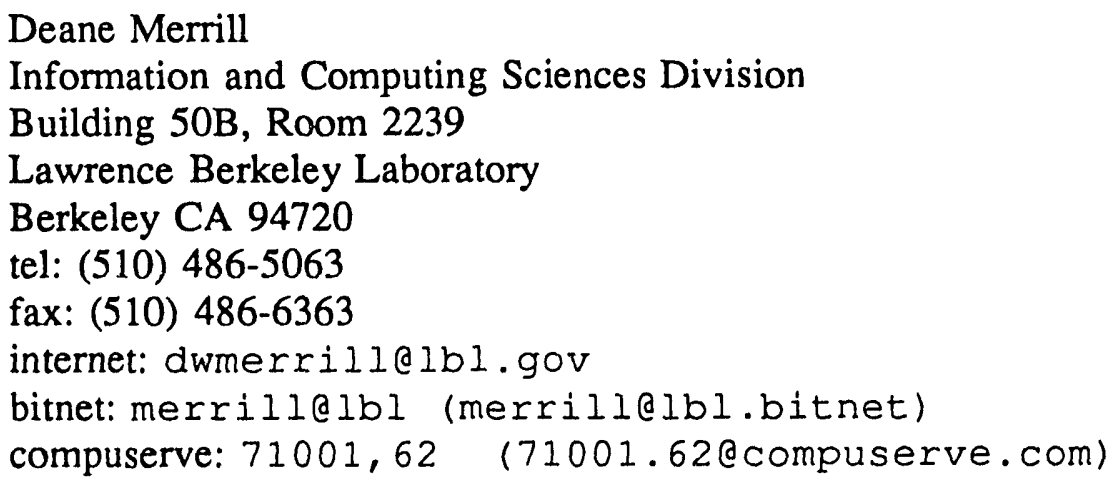

\section{OBTAINING THIS DOCUMENT}

This document is available via anonymous FTP from

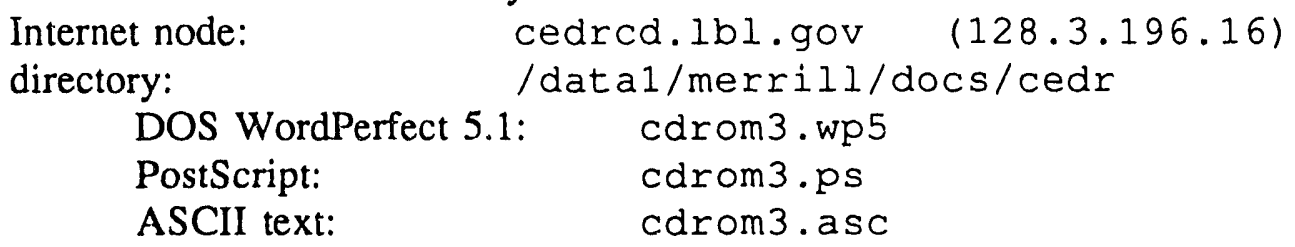

For example, to obtain cdrom3.ps for printing on your computer, move to a directory where you have write permission and then type:

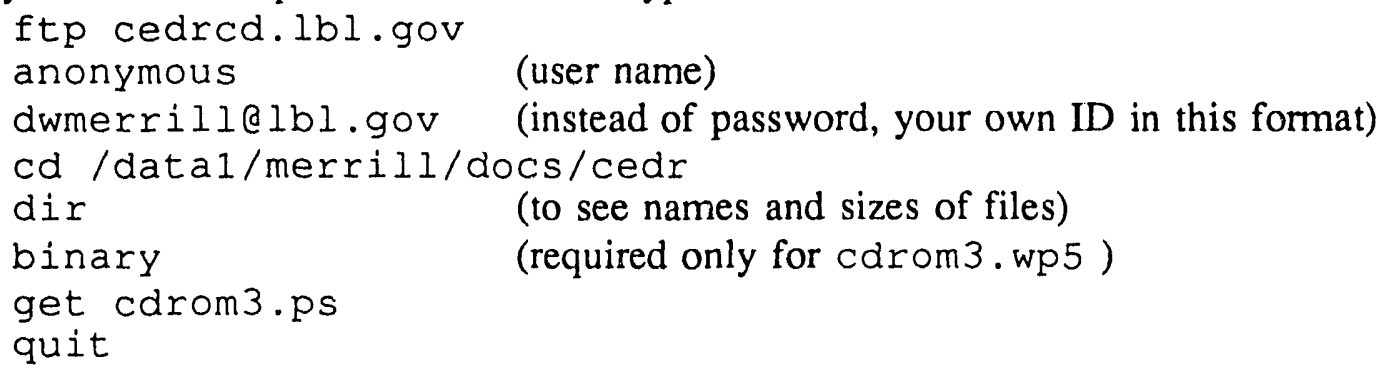

The same document in Macintosh Microsoft Word 5.0 format is in LBL AppleTalk zone icsd, Public Folder DWMerrill, file Census CD-ROM Data (Rev. 3).

Future revisions will be stored in the same locations and will be announced in the LBL Computing Newsletter.

This version is csr6.1bl.gov: c: \docs \cedr \cdrom3.49 $1 / 16 / 93$ 

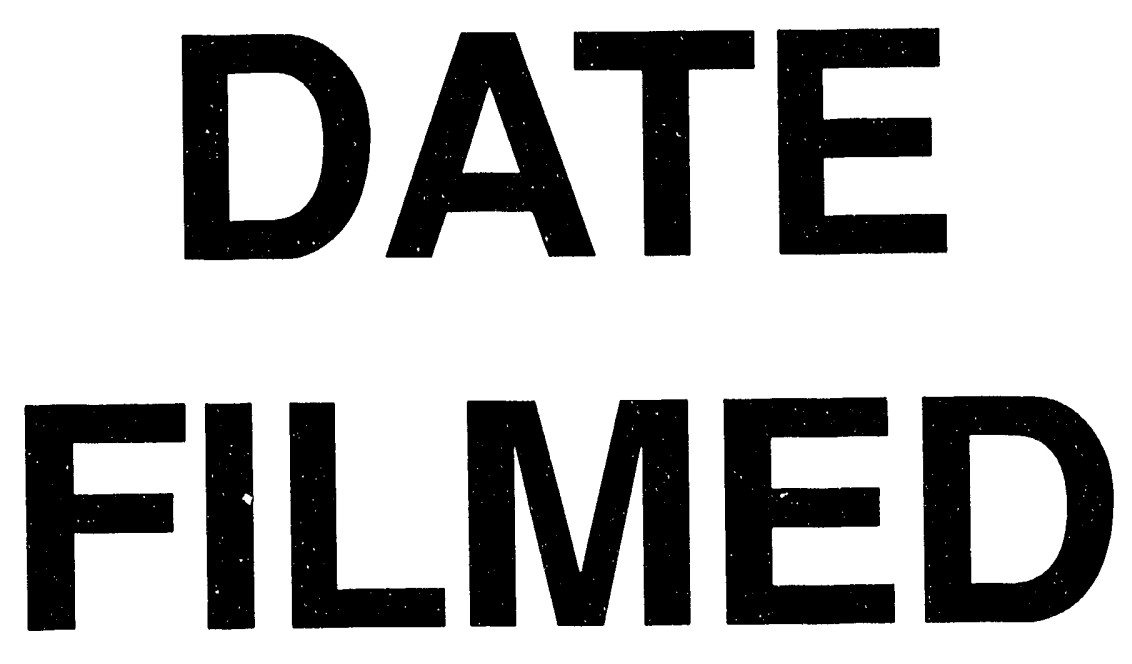

$$
7 / 27 / 93
$$
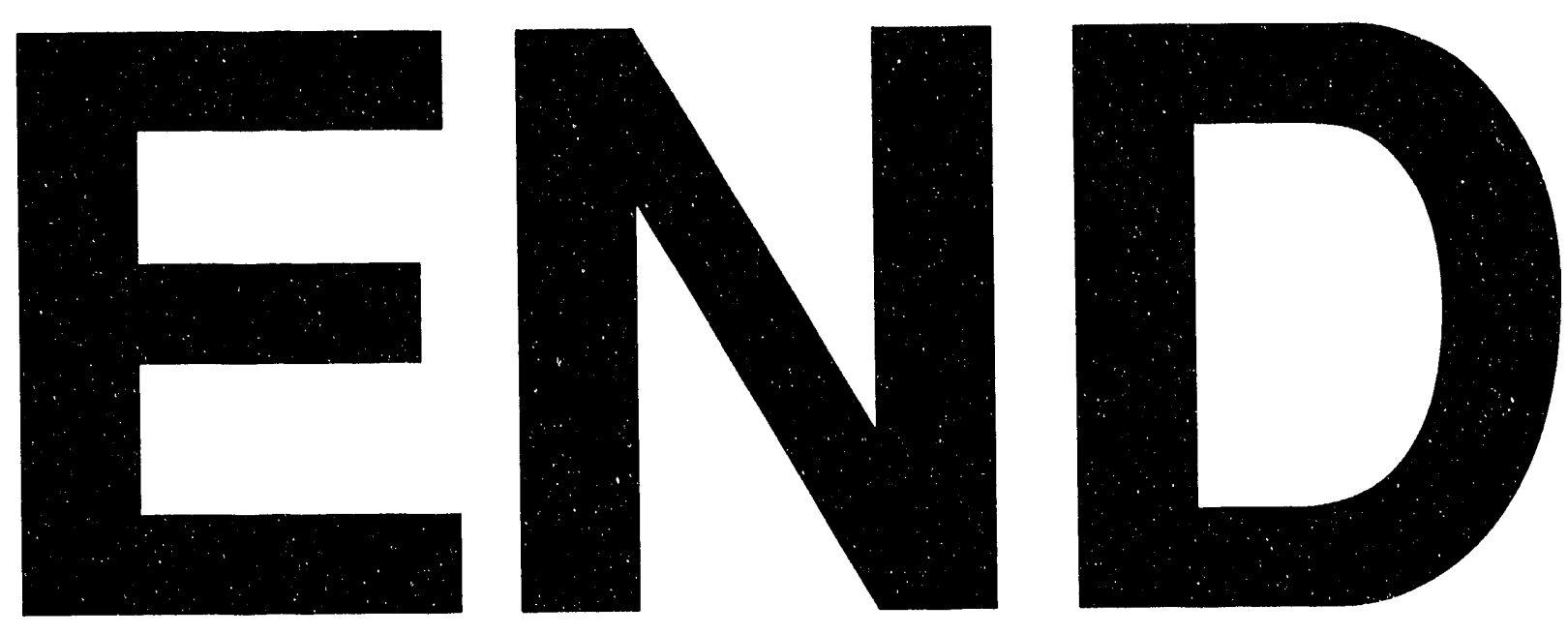
